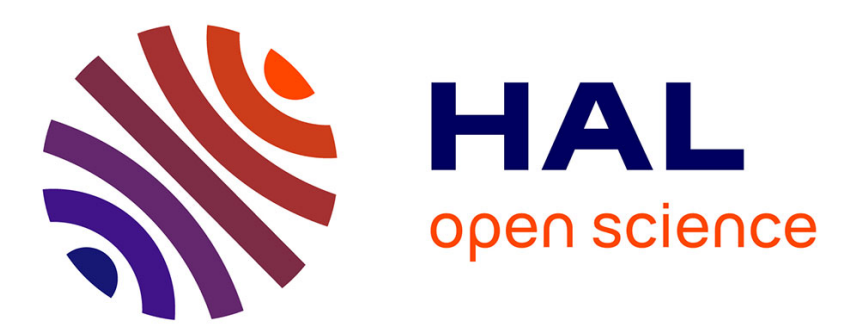

\title{
A numerical method for kinetic equations with discontinuous equations : application to mathematical modeling of cell dynamics
}

Benjamin Aymard, Frédérique Clément, Frédéric Coquel, Marie Postel

\section{To cite this version:}

Benjamin Aymard, Frédérique Clément, Frédéric Coquel, Marie Postel. A numerical method for kinetic equations with discontinuous equations : application to mathematical modeling of cell dynamics. SIAM Journal on Scientific Computing, 2013, 35 (6), 27 p. 10.1137/120904238 • hal-00751454v2

HAL Id: hal-00751454

https://hal.science/hal-00751454v2

Submitted on 7 Feb 2013

HAL is a multi-disciplinary open access archive for the deposit and dissemination of scientific research documents, whether they are published or not. The documents may come from teaching and research institutions in France or abroad, or from public or private research centers.
L'archive ouverte pluridisciplinaire HAL, est destinée au dépôt et à la diffusion de documents scientifiques de niveau recherche, publiés ou non, émanant des établissements d'enseignement et de recherche français ou étrangers, des laboratoires publics ou privés. 


\title{
A NUMERICAL METHOD FOR KINETIC EQUATIONS WITH DISCONTINUOUS EQUATIONS : APPLICATION TO MATHEMATICAL MODELING OF CELL DYNAMICS*
}

\author{
AYMARD BENJAMIN ${ }^{\dagger}$, CLÉMENT FRÉDÉRIQUE$^{\S}$, COQUEL FRÉDÉRIC ${ }^{\uparrow}$, AND POSTEL MARIE ${ }^{\dagger}$
}

\begin{abstract}
In this work, we propose a numerical method to handle discontinuous fluxes arising in transport-like equations. More precisely, we study hyperbolic PDEs with flux transmission conditions at interfaces between subdomains where coefficients are discontinuous. A dedicated finite volume scheme with a limited high order enhancement is adapted to treat the discontinuities arising at interfaces. The validation of the method is done on 1D and 2D toy problems for which exact solutions are available, allowing us to do a thorough convergence study. We then apply the method to a biological model focusing on complex cell dynamics, that initially motivated this study, and illustrates the full potentialities of the scheme.
\end{abstract}

Key words. kinetic equations, finite volumes, discontinuous coefficients, cell dynamics

AMS subject classifications. 65M08, 35L50, 35R05, 92C37

1. Introduction. This paper is dedicated to the study of first order equations with discontinuous flux functions in the space variable. Our generic setup is the broad field of coupling problems which has received considerable attention over the past decade. Let $\Omega \subset \mathbb{R}^{n}, n \geq 1$ be a simply connected open set, $\left\{\Omega_{i}\right\}_{i=1, \ldots, \mathcal{N}}$ a non overlapping collection of subsets of open subsets of $\Omega$, with $\cup_{i=1}^{\mathcal{N}} \overline{\Omega_{i}}=\bar{\Omega}$. Then consider the phase space $\mathcal{K} \subset \mathbb{R}^{F}$, with integer $F \geq 1$, a convex open set. For a given $T>0$, the problem reads: find $\phi:[0, T] \times \Omega \rightarrow \mathcal{K}$ solution of

$$
\begin{gathered}
\partial_{t} \phi+\operatorname{div}\left(f_{i}(\mathbf{x}, \phi)\right)=S_{i}(\mathbf{x}, \phi), \text { on }(0, T) \times \Omega_{i}, \quad \text { for all } i=1, \ldots, \mathcal{N}, \\
\phi(0, \mathbf{x})=\phi_{0}(\mathbf{x}), \text { on } \Omega \\
\phi(t, \mathbf{x})=0, \text { on }(0, T) \times \partial \Omega \\
\psi_{i, j}\left(\phi\left(t, \mathbf{x}^{-}\right)\right)=\phi\left(t, \mathbf{x}^{+}\right) \text {on }(0, T) \times \overline{\Omega_{i}} \cap \overline{\Omega_{j}},
\end{gathered}
$$

where $f_{i}: \Omega_{i} \times \mathcal{K} \rightarrow \mathbb{R}^{n \times F}$ and $S_{i}: \Omega_{i} \times \mathcal{K} \rightarrow \mathbb{R}^{F}$ are smooth functions. The last equation models the transmision condition between any pair of adjacent open sets $\Omega_{i}$ and $\Omega_{j}$, hence such that $\overline{\Omega_{i}} \cap \overline{\Omega_{j}} \neq \emptyset$, for $(i, j) \in\{1, \ldots, \mathcal{N}\}^{2}$. The transmission condition is prescribed thanks to the smooth function $\psi_{i, j}: \mathcal{K} \rightarrow \mathcal{K}$ which allows to define the right hand trace of $\phi\left(t, \mathbf{x}^{+}\right)$at $\overline{\Omega_{i}} \cap \overline{\Omega_{j}}$ from the left hand trace $\phi\left(t, \mathbf{x}^{-}\right)$.

This setup covers a wide range of applications besides mathematical modeling of cell proliferation which is our current interest here and will be detailed in the next paragraph: multiphase flow in porous media [7], traffic flow with discontinuous road surface conditions [10], sedimentation in thickener-clarifier units [9] for instance, which have generated a lot of interest and mathematical work in recent years. In a different domain of application, hydrodynamic limit for particle systems also lead to hyperbolic conservation laws with discontinuous fluxes with $f(x, \phi)=\lambda(x) h(\phi)$ as analyzed in [11].

In all these models, the mathematical difficulty lies in proving the well posedness of the IBVP problem, as it is well summarized in the review [8]. Already in the scalar case, namely with $F=1$, the $L^{1}$ contraction principle cannot be inferred from the Kruzkov's analysis [17], since it would require the Lipschitz continuity of the flux in the space variable.

${ }^{\dagger}$ UPMC UNIV PARIS 06, UMR 7598, LABORATOIRE JACQUES-LOUIS LIONS, F-75005, PARIS, FRANCE

${ }^{\ddagger}$ CNRS, UMR 7598, LABORATOIRE JACQUES-LOUIS LIONS, F-75005, PARIS, FRANCE

$\S$ CENTRE DE RECHERCHE INRIA PARIS-ROCQUENCOURT, DOMAINE DE VOLUCEAU, ROCQUENCOURT, B.P. 105 - F-78153 LE CHESNAY, FRANCE

${ }^{\top}$ CNRS AND CMAP, UMR 7641, ECOLE POLYTECHNIQUE, ROUTE DE SACLAY, F-91128 PALAISEAU CEDEX-FRANCE

*This work is part of the Inria Large Scale Initiative Action REGATE 
Besides classical waves, discontinuous solutions involve additional (standing) discontinuities. On the one hand, those additional waves may prevent the existence of an a priori BV bound for the solutions [1]. On the other hand, they also prevent classical entropy requirements from restoring uniqueness of the solution, even for the Riemann problem. Several distinct strengthened entropy conditions have been introduced in the literature and we refer for instance the reader to [1], [4], [11] and [8] for a review and exhaustive references. Distinct compactness frameworks based on these entropy conditions have then been proposed to prove well posedness of the problem (see again $[1,4,11,8]$ ).

Here we deal with a non conservative coupling setting where the velocities are nonlocal. As highlighted below, these two new mathematical features arise naturally in a biological context.

We address the model developed in [13] in the general context of cell dynamics, describing the time evolution of a density function depending on age and maturity variables $(n=2)$. This unknown is governed by a kinetic like equation involving velocities that are function of integro-moments of the unknown and the age and maturity variables. Closure equations for these velocities are naturally discontinuous in the age and maturity variables, precisely at biological checkpoints which correspond to the interfaces between the biological phases [22]. These discontinuities require additional information which are handled as local double initial boundary value problems (IBVP), where inner boundary conditions are formulated to express the biological switch.

The conservation hypothesis must clearly be abandoned: cell proliferation (through mitosis ${ }^{1}$ ) is modeled by a non conservative coupling and cell death (through apoptosis) also alters the global mass of the system. An additional specificity of the equation lies in its quasi linear integro differential nature. The flux functions $f_{i}$ are of the form

$$
f_{i}(\mathbf{x}, \phi)=u_{i}(\mathbf{x}, \phi) \phi, \quad \text { for } i=1, \ldots, \mathcal{N} .
$$

They arise from the weak dependence of the velocity field $u$ on the solution $\phi$ through a single moment $m_{f}(t)$

$$
u_{i}(\mathbf{x}, \phi)=v_{i}\left(\mathbf{x},\left\{m_{f}\right\}_{f=1, \ldots, F}\right), \quad \text { with } m_{f}(t)=\int_{\Omega} y \phi_{f}(t, \mathbf{x}) d \mathbf{x}, \quad \text { where } \mathbf{x}=(x, y) .
$$

More details on the biological background of the model will be provided in Section 5 which is devoted to its numerical simulation.

The theoretical study of this model has been done in [20]. The main difficulties come from the nonlocal velocity, the coupling between boundary conditions and the vector nature of the coupling problem $(F>1)$. Roughly speaking, the specific moment $m_{f}(t)$ of the solution entering the model is shown to exist as the fix point of a map from the continuous functions space. This leads to the existence and uniqueness of first a local weak solution, from which a global weak solution is then built. Various tools are at hand to compute the numerical approximation of this solution. Let us first mention that the general case of transport equations with discontinuous equations was first studied in a series of work by Bouchut and James [6] using duality arguments. Another standpoint is to address those discontinuities in terms of a coupling problem, as pioneered by Godlewski and Raviart [14]. We will adopt this later setting and show that it is well suited to our purpose. We will use techniques proposed in [14] and subsequent studies [3,7]. Here we pay special attention to the extension of these algorithms to higher order accuracy, which up to our knowledge, have not yet been addressed in 2D simulations for non conservative coupling and also barely in 2D in general. Although the well posedness issue is not handled with the same mathematical tools as in [20], the numerical methods that we have designed in this paper can be used in the application contexts cited above, like for instance traffic models [19].

Outline. From the numerical point of view we are focusing in this work on the discontinuous non conservative fluxes. The remaining of the paper is therefore organized in four sections. In Section 2, we describe toy models consisting of linear hyperbolic PDE with piecewise constant speeds and linear source term, in 1D and 2D. In section 3 , we present the numerical scheme, first recalling the Finite Volume scheme and then specifically dealing with the transmission conditions. For piecewise constant speed linear transport with or without linear source term, we design a

\footnotetext{
${ }^{1}$ Process in cell proliferation through which a mother cell gives birth to two daughter cells
} 
third order scheme by combining a limited reconstruction in space and a Runge Kutta time quadrature. We describe in details the numerical scheme at the interfaces where the speed and source coefficients are discontinuous, with or without discontinuity of the flux, in order to maintain the precision of the overall computation.

The numerical validation is performed in Section 4 on several 1D and 2D test cases and based on a thorough convergence study. Whenever it is possible, the exact solutions are used as references (the derivation of the analytical solutions is postponed to the Appendix). Finally, we present in Section 5 the biological model describing the cell dynamics in developing ovarian follicles [13] which has motivated our work and perform simulations that validate the numerical method for this application. If this model has already been subject to numerical simulations based on finite volumes (see $[12,13]$ for instance), the first rigorous numerical test was performed recently in a preliminary study [5], that only dealt with low-order treatment of discontinuities. The novelty here is the high order enhancement at discontinuous interfaces, which is a particularly challenging question.

2. Toy models. In this section we present generic toy models with piecewise constant coefficients for which exact solutions are available, and which we will use to validate the numerical method. The weak nonlinearity arising in biological models from the dependence of the speeds and source term on the moments of the solution is left out. It is therefore sufficient to consider a simplified scalar model defined for $(x, y) \in \Omega=\mathbb{R}^{2}, t>0$.

$$
\left\{\begin{array}{l}
\frac{\partial \phi(x, y, t)}{\partial t}+\frac{\partial(g(x, y) \phi(x, y, t))}{\partial x}+\frac{\partial(h(x, y) \phi(x, y, t))}{\partial y}=-\Lambda(x, y) \phi(x, y, t) \\
\phi(x, y, 0)=\phi_{0}(x, y) \quad \text { (initial condition) } \\
\left.\psi_{L}\left(g\left(x_{s}^{-}, y\right) \phi\left(x_{s}^{-}, y, t\right)\right)=g\left(x_{s}^{+}, y\right) \phi\left(x_{s}^{+}, y, t\right) \quad \text { (flux cond. on } \overline{\Omega_{1}} \cap \overline{\Omega_{2}}\right) \\
\left.\psi_{B}\left(h\left(x, y_{s}^{-}\right) \phi\left(x, y_{s}^{-}, t\right)\right)=h\left(x, y_{s}^{+}\right) \phi\left(x, y_{s}^{+}, t\right) \quad \text { (flux cond. on } \overline{\Omega_{i}} \cap \overline{\Omega_{3}}, \text { for } i=1,2\right)
\end{array}\right.
$$

with the piecewise constant velocities $g(x, y)$ and $h(x, y)$ and source term $\Lambda(x, y)$ defined in the subregions depicted on Figure 2.1, namely $\Omega_{1}=\left\{(x, y) \in \mathbb{R}^{2}, x<x_{s}, y<y_{s}\right\}, \Omega_{2}=\left\{(x, y) \in \mathbb{R}^{2}, x>x_{s}, y<y_{s}\right\}$ and $\Omega_{3}=\{(x, y) \in$ $\left.\mathbb{R}^{2}, y>y_{s}\right\}$. To fix ideas, we can already assume that $\phi(x, y)$ represents a density of cells, and that its integral over $\Omega$ is the total mass of the system. Furthermore, thanks to the simplicity of the geometry, we can use a cartesian grid discretization and a numerical scheme deduced from the $1 \mathrm{D}$ problem by tensorization. We will therefore describe the numerical method on the following $1 \mathrm{D}$ toy problem

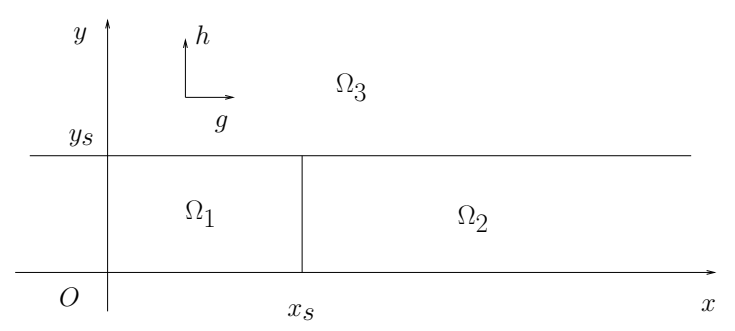

FIGURE 2.1. Spatial set-up for the $2 D$ toy model. $g$ and $h$ respectively denote the velocities in the $x$ and $y$ directions.

$$
\left\{\begin{array}{l}
\partial_{t} \phi(x, t)+\partial_{x} g(x) \phi(x, t)=-\Lambda(x) \phi(x, t) \quad \text { for } x \in \mathbb{R}, t>0 \\
\phi(x, 0)=\phi_{0}(x) \quad \text { (initial condition) } \\
\psi_{L}\left(g\left(x_{s}^{-}\right) \phi\left(x_{s}^{-}, t\right)\right)=g\left(x_{s}^{+}\right) \phi\left(x_{s}^{+}, t\right) \quad \text { (flux condition) }
\end{array}\right.
$$

with

$$
g(x)=\left\{\begin{array}{ll}
g_{L}, & \text { for } x<x_{s}, \\
g_{R} & \text { elsewhere }
\end{array} \quad \Lambda(x)=\left\{\begin{array}{cc}
\Lambda_{L}, & \text { for } x<x_{s} \\
\Lambda_{R} & \text { elsewhere }
\end{array}\right.\right.
$$


In order to deal with all the situations encountered in the real-life problem to be addressed in section 5, the transmission conditions in (2.2) will be in turn

$$
\begin{aligned}
& \psi_{L}(z)=z \quad \text { (flux continuity between left and right zones) } \\
& \psi_{L}(z)=2 z \quad \text { (doubling of flux between left and right zones) } \\
& \psi_{L}(z)=0 \quad \text { (waterproof interface between left and right zones) }
\end{aligned}
$$

and the source term will be in turn

$$
\begin{array}{ll}
\Lambda_{L}(x)=\Lambda_{R}=0 & \text { (no source) } \\
\Lambda_{L}(x)=1, \Lambda_{R}=0 & \text { (source in left zone only) } \\
\Lambda_{L}(x)=0, \Lambda_{R}=1 & \text { (source in right zone only) }
\end{array}
$$

3. Numerical scheme. We now turn to the numerical method. We first recall the finite volume scheme in the continuous regions, and then present the method that we have designed to deal with the transmission conditions.

3.1. Discretization. In this paragraph we describe the numerical scheme for the simplified problems (2.2) and (2.1). To solve (2.1) we restrict the computational domain to $\left[0, L_{x}\right] \times\left[0, L_{y}\right]$, enforcing periodical boundary conditions on the outer boundaries. We set the domain dimensions as $L_{x}=L_{y}=1$. We denote by $N_{x}$ the number of grid meshes in each direction and by $\Delta x=L_{x} / N_{x}$ the space step. The step needs to be chosen so that the locations of the interfaces $x_{s}$ and $y_{s}$, where the speed coefficients $g(x, y)$ and $h(x, y)$ are discontinuous, fall on grid points. From now on we focus on the $1 \mathrm{D}$ finite volume scheme to solve (2.2), since the $2 \mathrm{D}$ scheme can be obtained by tensorization. Along the spatial grid

$$
x_{k}=k \Delta x, \quad x_{k+1 / 2}=(k+1 / 2) \Delta x, \text { for } k=0, \ldots, N_{x},
$$

the time discretization is defined by

$$
t^{0}=0, \quad t^{n+1}=t^{n}+\Delta t^{n}, \quad \text { for } n=0, \ldots, N
$$

with $N$ such that $t^{N}=t_{\text {final }}$, and time steps $\Delta t^{n}$ that may change at each iteration, in order to preserve stability. The unknowns are the approximate mean values of the solution in each grid mesh $\left[x_{k}, x_{k+1}\right]$

$$
\phi_{k}^{n} \approx \frac{1}{\Delta x} \int_{x_{k}}^{x_{k+1}} \phi\left(x, t^{n}\right) d x . \quad \text { for } \quad k=0, \ldots, N_{x}-1 .
$$

Equation (2.2) is then explicitly discretized to obtain a recursion formula

$$
\phi_{k}^{n+1}=\phi_{k}^{n}-\frac{\Delta t^{n}}{\Delta x}\left(F_{k+1}\left(\phi^{n}\right)-F_{k}\left(\phi^{n}\right)\right)-\Delta t^{n} \Lambda\left(x_{k+\frac{1}{2}}\right) \phi_{k}^{n}
$$

where $F_{k}$ is the numerical flux across $x_{k}$, designed using a limiter strategy. Indeed, it is well known that first order schemes, like the Godunov scheme, are diffusive, and that second order schemes, like Lax Wendroff scheme, generate oscillations in the neighborhood of discontinuities. In order to get a stable as well as precise scheme, we take a weighting of a low order scheme and high order scheme, and we define the limited numerical flux

$$
F_{k}=F_{k}^{L o w}+\ell\left(r_{k}\right)\left(F_{k}^{H i g h}-F_{k}^{L o w}\right),
$$

where $\ell$ is the limiter function designed by Koren [16]

$$
\ell_{\text {Koren }}(r)=\max \left(0, \min \left(2 r, \frac{2+r}{3}, 2\right)\right),
$$


and $r_{k}$ is defined by

$$
\begin{aligned}
& r_{k}=\mathcal{R}\left(\left(g_{k+i} \phi_{k+i}\right)_{i=-2, \ldots, 1} ;\left(g_{k+i}\right)_{i=-2, \ldots, 1}\right) \\
& \mathcal{R}\left(\left(z_{k+i}\right)_{i=-2, \ldots, 1} ;\left(g_{k+i}\right)_{i=-2, \ldots, 1}\right)=\left\{\begin{array}{l}
\frac{z_{k-1}-z_{k-2}}{z_{k}-z_{k-1}} \quad \text { if } \quad g_{k+i} \geq 0 \forall i=-2, \ldots, 1 \\
\frac{z_{k+1}-z_{k}}{z_{k}-z_{k-1}} \text { if } \quad g_{k+i} \leq 0 \quad \forall i=-2, \ldots, 1 \\
0 \text { otherwise, }
\end{array}\right.
\end{aligned}
$$

with the notation $g_{k}=g\left(x_{k}\right)$. This ratio is a good indicator of the regularity of the function (see [21]). In fact, a steep gradient or a discontinuity gives a ratio far from 1, whereas a smooth function gives a ratio close to 1 .

The first order flux entering equation (3.4) is the Godunov flux

$$
F_{k}^{\text {Low }}\left(\phi^{n}\right)=\left(g_{k-1}^{n}\right)^{+} \phi_{k-1}^{n}+\left(g_{k}^{n}\right)^{-} \phi_{k}^{n},
$$

and the high order flux is the Lax Wendroff one, which is of second order in space wherever the function $g(x)$ is continuous.

$$
F_{k}^{H i g h}\left(\phi^{n}\right)=\frac{1}{2}\left(g_{k-1}^{n} \phi_{k-1}^{n}+g_{k}^{n} \phi_{k}^{n}\right)
$$

The CFL stability condition is

$$
\Delta t^{n} \leq \min \left(C F L \frac{\Delta x}{\max _{k}\left|g_{k}\right|}, \frac{1}{\max _{k}\left|\Lambda_{k+\frac{1}{2}}\right|}\right)
$$

with $C F L \leq \frac{1}{2}$ and the notation $\Lambda_{k+1 / 2}=\Lambda\left(x_{k+1 / 2}\right)$.

Choosing Koren limiter in (3.4) provides third order in space for the convective part of the equation in each domain where it is continuously defined. The source term being discretized by a center point quadrature is at most second order in each domain. Second order in time (third order when $\Lambda=0$ ) is achieved by a third order Runge-Kutta method ([15])

$$
\begin{aligned}
\mathcal{B}_{k}^{1} & =F_{k+1}\left(\phi^{n}\right)-F_{k}\left(\phi^{n}\right)+\Delta x \Lambda_{k+1 / 2} \phi_{k}^{n}, \\
\phi_{k}^{*} & =\phi_{k}^{n}-\frac{\Delta t^{n}}{\Delta x} \mathcal{B}_{k}^{1}, \\
\mathcal{B}_{k}^{2} & =F_{k+1}\left(\phi^{*}\right)-F_{k}\left(\phi^{*}\right)+\Delta x \Lambda_{k+1 / 2} \phi_{k}^{*}, \\
\phi_{k}^{* *} & =\phi_{k}^{n}-\frac{1}{4} \frac{\Delta t^{n}}{\Delta x} \mathcal{B}_{k}^{1}-\frac{1}{4} \frac{\Delta t^{n}}{\Delta x} \mathcal{B}_{k}^{2} \\
\mathcal{B}_{k}^{3} & =F_{k+1}\left(\phi^{* *}\right)-F_{k}\left(\phi^{* *}\right)+\Delta x \Lambda_{k+1 / 2} \phi_{k}^{* *}, \\
\phi_{k}^{n+1} & =\phi_{k}^{n}-\frac{1}{6} \frac{\Delta t^{n}}{\Delta x} \mathcal{B}_{k}^{1}-\frac{1}{6} \frac{\Delta t^{n}}{\Delta x} \mathcal{B}_{k}^{2}-\frac{2}{3} \frac{\Delta t^{n}}{\Delta x} \mathcal{B}_{k}^{3},
\end{aligned}
$$

We will now focus on the interface between two subdomains where function $g$ can be discontinuous, neglecting the source term for the time being.

3.2. Treatment of discontinuous coefficients. The domain is discretized in such a manner that the interface between two subregions where $g$ is continuous is placed on an edge between two grid meshes. Let us consider that the interface position $x_{s}=x_{K}$ is at the interface between meshes $\left[x_{K-1}, x_{K}\right]$ and $\left[x_{K}, x_{K+1}\right]$ as depicted on Figure 3.1. To adapt the finite volume scheme designed in the previous paragraph so that it can handle the transmission condition, we define for each interface at grid point $x_{k}$ two fluxes: the left flux $F_{k}^{L}$ and the right flux $F_{k}^{R}$ (Figure 3.1). Then we rewrite equation (3.3) as

$$
\phi_{k}^{n+1}=\phi_{k}^{n}-\frac{\Delta_{t}^{n}}{\Delta_{x}}\left(F_{k+1}^{L}-F_{k}^{R}\right)
$$




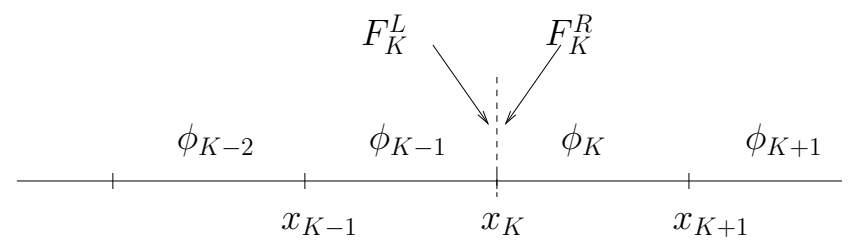

FIGURE 3.1. Left and right flux surrounding the interface between meshes $\left[x_{K-1}, x_{K}\right]$ and $\left[x_{K}, x_{K+1}\right]$.

If there is no transmission condition on the flux, we have

$$
F_{k}^{L}=F_{k}^{R}=F_{k}
$$

defined by (3.4). In contrast, if there is a transmission condition such as (2.5), we set

$$
F_{K}^{R}=\psi_{L}\left(F_{K}^{L}\right) .
$$

The value of the numerical fluxes defined by (3.7) and (3.8) are derived for smooth coefficients $g(x)$ and continuous fluxes $g(x) \phi(x)$. They have therefore to account for the transmission condition $\psi_{L}$, seen from the left side of $x_{s}$. We define, at the interface $K$, the first order flux

$$
F_{K}^{\text {Low, } L}\left(\phi^{n}\right)=\left(g_{K-1}^{n}\right)^{+} \phi_{K-1}^{n}+\frac{1}{2}\left(1-\operatorname{sign}\left(g_{K}^{n}\right)\right) \psi_{L}^{-1}\left(g_{K}^{n} \phi_{K}^{n}\right),
$$

and the second order flux

$$
F_{K}^{H i g h, L}\left(\phi^{n}\right)=\frac{1}{2}\left(g_{K-1}^{n} \phi_{K-1}^{n}+\psi_{L}^{-1}\left(g_{K}^{n} \phi_{K}^{n}\right)\right) .
$$

Furthermore, the high order enhancement using a limited combination such as (3.4) is affected by the presence of an interface not only at $x_{K}$ but also in its vicinity. Indeed, if the velocity $g(x)$ is positive, the limiter "sees" interface $K$ at neighboring interfaces $k=K-1, \ldots, K+1$ through the ratio $r_{k}$. The transmission condition (3.10) causes the ratio $r_{k}$ (3.5) to depart from 1 on these interfaces and consequently induces a loss of accuracy in the numerical scheme. This can be avoided by computing the ratio $r_{k}$ from the continuous quantities seen from $x_{k}$. Namely, at interfaces $K-1, K, K+1$, the limiter is computed using

$$
\left\{\begin{array}{l}
r_{K-1}=\mathcal{R}\left(g_{K-3} \phi_{K-3}, g_{K-2} \phi_{K-2}, g_{K-1} \phi_{K-1}, \psi_{L}^{-1}\left(g_{K} \phi_{K}\right) ;\left(g_{K+i}\right)_{i=-3, \ldots, 0}\right) \\
r_{K}=\mathcal{R}\left(\psi_{L}\left(g_{K-2} \phi_{K-2}\right), \psi_{L}\left(g_{K-1} \phi_{K-1}\right), g_{k} \phi_{K}, g_{K+1} \phi_{K+1} ;\left(g_{K+i}\right)_{i=-2, \ldots, 1}\right) \\
r_{K+1}=\mathcal{R}\left(\psi_{L}\left(g_{K-1} \phi_{K-1}\right), g_{K} \phi_{K}, g_{K+1} \phi_{K+1}, g_{K+2} \phi_{K+2} ;\left(g_{K+i}\right)_{i=-1, \ldots, 2}\right)
\end{array}\right.
$$

instead of (3.5).

To fix ideas, we apply this method in cases (2.4) and (2.5) encountered in our application model.

3.2.1. First application: Flux continuity with discontinuous speed. In this case the transmission condition is

$$
\psi_{L}=I d .
$$

At interface $K$, the condition is

$$
(g \phi)_{K}^{R}=(g \phi)_{K}^{L}
$$

with

$$
g_{K-1} \neq g_{K} \text {. }
$$

The transmission condition (3.10) provides the flux from the right side of the interface

$$
F_{K}^{R}=F_{K}^{L} .
$$

The scheme works well without modification, both at first and third order. 
3.2.2. Second application: Doubling flux. In this case the transmission condition (3.10) is

$$
\psi_{L}(\mathcal{F})=2 \mathcal{F} .
$$

At interface $K$, the condition is

$$
(g \phi)_{K}^{R}=2(g \phi)_{K}^{L},
$$

with

$$
g_{K-1} \neq g_{K}
$$

The flux on the left of the interface is computed using continuous values in the definitions of (3.7) and (3.8). From the left side, the continuous values are $g \phi$ for $x<x_{K}$ and $\psi_{L}(g \phi)$ for $x>x_{K}$. Therefore we write for the first order flux

$$
F_{K}^{l o w, L}=\left(g_{K-1}\right)^{+} \phi_{K-1}+\frac{\left(g_{K}\right)^{-} \phi_{K}}{2},
$$

and for the second order flux

$$
F_{K}^{h i g h, L}=\frac{g_{K-1} \phi_{K-1}+\frac{g_{K} \phi_{K}}{2}}{2} .
$$

Concerning the limiter, it has to take into account the doubling condition on three meshes in the vicinity of the interface. At interfaces $K-1, K, K+1$ the limiter (3.13) is computed from the continuous quantities

$$
\left\{\begin{array}{l}
r_{K-1}=\mathcal{R}\left(g_{K-3} \phi_{K-3}, g_{K-2} \phi_{K-2}, g_{K-1} \phi_{K-1}, \frac{g_{K} \phi_{K}}{2} ;\left(g_{K+i}\right)_{i=-3, \ldots, 0}\right), \\
r_{K}=\mathcal{R}\left(2 g_{K-2} \phi_{K-2}, 2 g_{K-1} \phi_{K-1}, g_{K} \phi_{K}, g_{K+1} \phi_{K+1} ;\left(g_{K+i}\right)_{i=-2, \ldots, 1}\right), \\
r_{K+1}=\mathcal{R}\left(2 g_{K-1} \phi_{K-1}, g_{K} \phi_{K}, g_{K+1} \phi_{K+1}, g_{K+2} \phi_{K+2} ;\left(g_{K+i}\right)_{i=-1, \ldots, 2}\right) .
\end{array}\right.
$$

The transmission condition (3.10) provides the flux from the right side of the interface

$$
F_{K}^{R}=2 F_{K}^{L}
$$

4. Numerical validation. In the sequel, we present several numerical simulations to substantiate the validation of our method. Two 1D situations are handled, one with a flux continuity (2.4), another with a doubling at the interface (2.5), with or without a source term (2.7), (2.8), (2.9). We then present two 2D test cases: a shear phenomenon which is encountered between zones $\Omega_{1}$ and $\Omega_{3}$, and a waterproof condition (2.6), between zones $\Omega_{2}$ and $\Omega_{3}$.

4.1. 1D test cases. For this set of test cases we can compare the numerical solution at final time $t^{N}$ with the exact solution described in Appendix A.1 and compute the L1-norm relative error

$$
E_{\Delta x}=\frac{\sum_{k=0}^{N x}\left|\phi_{k}^{N}-\bar{\phi}_{k}^{N}\right|}{\sum_{k=0}^{N x}\left|\bar{\phi}_{k}^{N}\right|}
$$

where $\bar{\phi}_{k}^{N}$ is the mean value of the exact solution at final time on mesh $\left[x_{k}, x_{k+1}\right]$. This mean value is itself estimated with a quadrature formula - the second order central point formula. This is actually justified for the 1D test cases where the discontinuity of the solution coincides with a grid point. In order to recover the expected behavior the initial condition is a smooth gaussian function of total mass equal to 1 and centered on $c_{x}=0.3$.

$$
\phi_{0}(x)=\frac{1}{2 \pi \sigma} \exp \left(\frac{1}{2}\left(\frac{\left(x-c_{x}\right)^{2}}{\sigma^{2}}\right)\right) .
$$


B. Aymard, F. Clément, F. Coquel and M. Postel

\begin{tabular}{|c|c|c|c|c|c|c|c|c|}
\hline test & description & $\psi_{L}(\mathcal{F})$ & $g_{L}$ & $g_{R}$ & $\Lambda_{L}$ & $\Lambda_{R}$ & $\mathrm{p}$ & $E .10^{4}$ \\
\hline 1 & $\begin{array}{l}\text { Continuous flux, speed } \\
\text { discontinuity on } \Omega_{1}-\Omega_{2} \\
\text { interface, no source }\end{array}$ & $\mathcal{F}$ & $\begin{array}{c}0.5 \\
1 \\
2 \\
3\end{array}$ & 1 & 0 & 0 & $\begin{array}{l}2.35 \\
2.39 \\
2.40 \\
2.29\end{array}$ & $\begin{array}{l}9.23 \\
9.39 \\
9.74 \\
9.71\end{array}$ \\
\hline 2 & $\begin{array}{l}\text { Doubling flux, speed } \\
\text { discontinuity on } \Omega_{1}-\Omega_{2} \\
\text { interface, no source }\end{array}$ & $2 \mathcal{F}$ & 1 & $\begin{array}{c}0.5 \\
1 \\
2 \\
3\end{array}$ & 0 & 0 & $\begin{array}{l}2.40 \\
2.38 \\
2.35 \\
2.34\end{array}$ & $\begin{array}{c}10.25 \\
9.81 \\
9.64 \\
9.61\end{array}$ \\
\hline 3 & $\begin{array}{l}\text { Continuous flux, speed } \\
\text { discontinuity on } \Omega_{1}-\Omega_{2} \\
\text { interface, source in } \Omega_{1}\end{array}$ & $\mathcal{F}$ & $\begin{array}{c}0.5 \\
1 \\
2 \\
3\end{array}$ & 1 & 1 & 0 & $\begin{array}{l}2.35 \\
2.39 \\
2.37 \\
2.28\end{array}$ & $\begin{array}{l}8.89 \\
9.22 \\
9.47 \\
9.63\end{array}$ \\
\hline 4 & $\begin{array}{l}\text { Doubling flux, speed } \\
\text { discontinuity on } \Omega_{1}-\Omega_{2} \\
\text { interface, source in } \Omega_{1}\end{array}$ & $2 \mathcal{F}$ & 1 & $\begin{array}{c}0.5 \\
1 \\
2 \\
3\end{array}$ & 0 & 1 & $\begin{array}{l}2.42 \\
2.36 \\
2.34 \\
2.33\end{array}$ & $\begin{array}{c}10.34 \\
9.68 \\
9.60 \\
9.58\end{array}$ \\
\hline
\end{tabular}

$1 D$ test cases description, asymptotic order $p$ of the L1-norm error $O\left(\Delta x^{p}\right)$ and relative error value for $\Delta x=10^{-3}$. Each test case is run for four different speed contrasts, leading to slightly different asymptotic behavior.

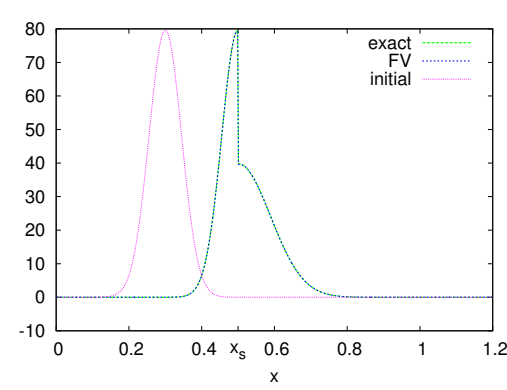

a) $g_{L}=0.5$ (speed up after $\left.x_{s}=0.5\right)$

$t^{N}=0.4$

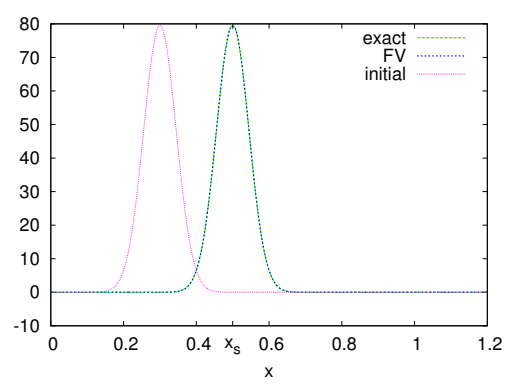

b) $g_{L}=1$ (no velocity jump) $t^{N}=0.2$

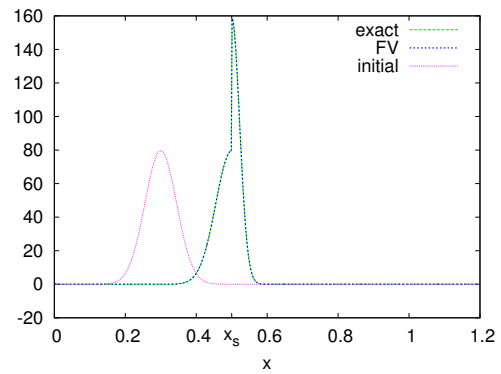

c) $g_{L}=2\left(\right.$ slow down after $\left.x_{s}=0.5\right)$

$t^{N}=0.1$

FIGURE 4.1. $1 D$ test case 1: flux continuity condition and velocity jump at interface $x_{s}=0.5$. Snapshot of the density $\phi(x, t)$ at initial time and when it crosses the interface between zones $\Omega_{1}$ and $\Omega_{2}\left(t=t^{N}\right)$.

Test case 1. Continuous flux, speed discontinuity on $\Omega_{1}-\Omega_{2}$ interface, no source. This tests mimics what happens at the transition (2.4) between zones $\Omega_{1}$ and $\Omega_{2}$. The speed is equal to 1 in the second half of the domain, and takes values 0.5, 1 (for reference), 2 or 3 in the first half of the domain. As described in Appendix A.1 the exact solution in that case is

$$
\phi^{\text {exact }}(x, t)=\left\{\begin{array}{l}
\phi_{0}\left(x-g_{L} t\right) \text { for } x<x_{s}, \\
g_{L} \phi_{0}\left(x_{s}-g_{L}\left(t-x-x_{s}\right)\right) \text { for } x_{s}-t \leq x-t<x_{s}, \\
\phi_{0}(x-t) \text { for } x-t \geq x_{s}
\end{array}\right.
$$

In Figure 4.1, we plot the density $\phi$ at a time $t^{N}=0.2 / g_{L}$, chosen so that half the mass has already crossed the interface between zones $\Omega_{1}$ and $\Omega_{2}$. Since the flux is continuous at $x_{s}=0.5$ and the speed is discontinuous, the solution presents a discontinuity (except for the special case $g_{L}=1$ ). We can notice the good quality of the approximation, compared to the exact solution. There are no oscillations in the neighborhood of the discontinuity, neither numerical diffusion. Remark that if the speed decreases much on the right side of the interface, the density shape sharpens. This is qualitatively similar to what happens for a traffic flow when there is a sudden speed limitation. This phenomenon penalizes the accuracy. In panel b) of Figure 4.2, the $L 1$-norm error with respect to the space step $\Delta x$ is displayed in logarithmic scale, which 
allows us to compute the convergence rate. Depending on the precise value of $g_{L}$, the convergence rate varies between 2.26 and 2.4. More precisely, it gets worse as the value of $g_{L}$ increases. This can be explained by looking at the contrast between $g_{L}$ and $g_{R}=1$. By analogy with the traffic flow, the interface between a high speed on the left and a lower one on the right hand side corresponds to a sudden speed decrease which generates an accumulation of traffic. The density profile becomes narrower and it becomes harder to make accurate simulations. In contrast, when the speed is higher on the right, the density profile becomes smoother and it becomes easier to approximate the solution. In any case, the best order of convergence 2.4 is achieved when $g_{L}=g_{R}=1$. In panel a) of Figure 4.2, the total mass

$$
m_{0}(t)=\int_{0}^{L_{x}} \phi(x, t) d x
$$

is displayed with respect to the time to check whether the scheme is conservative. We also perform a 2D generalization

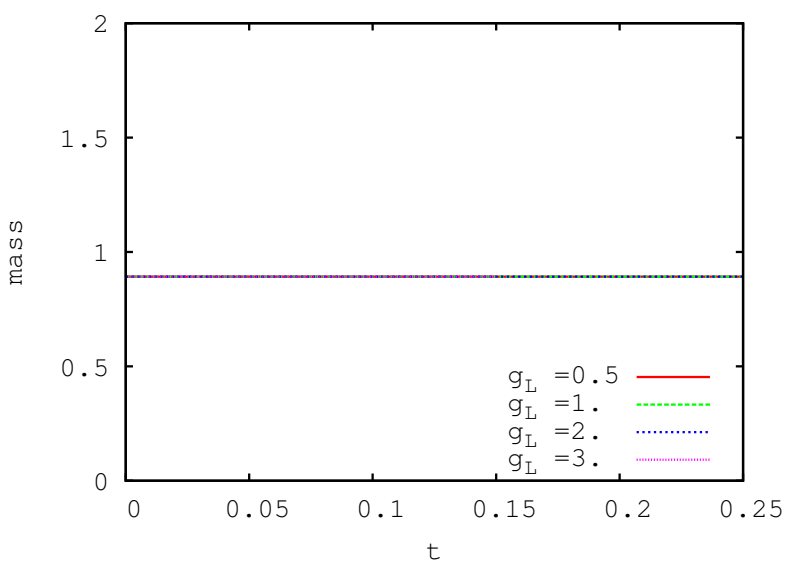

a) Mass with respect to time

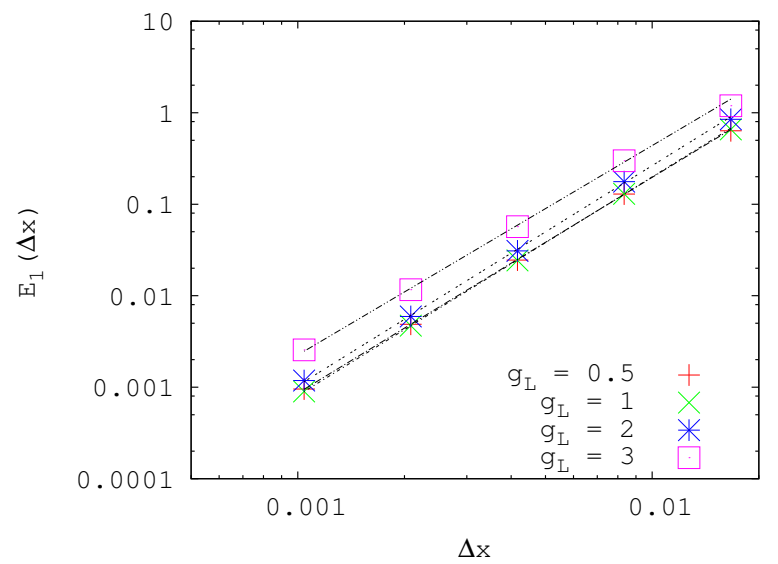

b) Error convergence with respect to $\Delta x$

FIGURE 4.2. $1 D$ test case 1: flux continuity condition and velocity jump at interface $x_{s}=0.5$. In panel a) the total mass remains constant with time. In panel $b$ ) the $L_{1}$-norm error goes to 0 with $\Delta x$. The convergence rates (around 2.4) are gathered in Table 4.1.

of this test in the case $g_{L}=2$, with a smooth initial condition

$$
\phi_{0}(x, y)=\frac{1}{2 \pi \sigma^{2}} \exp \left(\frac{1}{2}\left(\frac{\left(x-c_{x}\right)^{2}}{\sigma^{2}}+\frac{\left(y-c_{y}\right)^{2}}{\sigma^{2}}\right)\right) .
$$

centered on $\left(c_{x}, c_{y}\right)=(0.3,0.15)$ and the same variance $\sigma^{2}=0.002$ as before. The corresponding results are displayed on Figure 4.3.

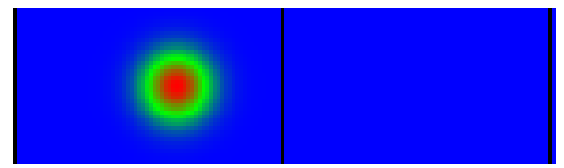

a) Initial time. The density bump lies in zone $\Omega_{1}$.

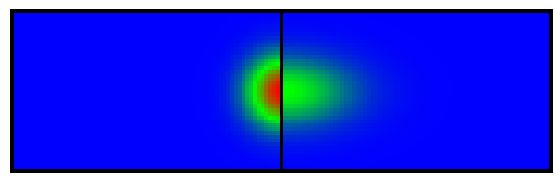

b) Intermediate time $t=0.15$.

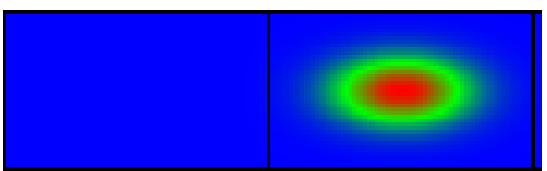

c) Final time $t=0.25$. The density bump lies in zone $\Omega_{2}$.

FIGURE 4.3. $2 D$ visualization of test case 1 : flux continuity condition and velocity jump at interface $x_{s}=0.5, u_{L}=0.5, u_{R}=1$. Snapshot of the density (initial time, passing interface, final time). (CFL $=0.4, \Delta x=0.001$ ).

Test case 2. Doubling flux, speed discontinuity on $\Omega_{1}-\Omega_{2}$ interface, no source. The second test case addresses flux discontinuity described in paragraph 3.2.2. The speed is equal to $g_{L}=1$ for $x<x_{s}$ and takes successively the 

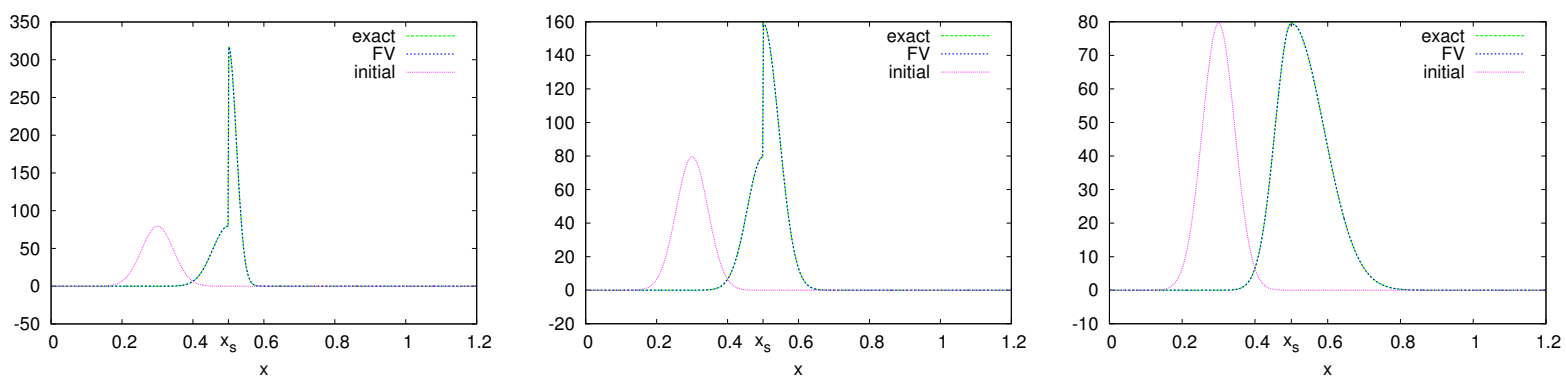

a) $g_{R}=0.5$

b) $g_{R}=1$ (no velocity jump)

c) $g_{R}=2$ (no density jump)

FIGURE 4.4. $1 D$ test case 2: doubling flux condition and velocity jump at interface $x_{s}=0.5$. Snapshot of the density $\phi(x, t)$ at initial time and when it crosses the interface between zones $\Omega_{1}$ and $\Omega_{2}(t=0.2)$.

values $g_{R}=0.5,1,2$ or 3 for $x>x_{s}$. This time the exact solution is

$$
\phi^{\text {exact }}(x, t)=\left\{\begin{array}{l}
\phi_{0}(x-t) \quad \text { for } x<x_{s}, \\
\frac{2}{g_{R}}\left(\phi_{0}\left(x_{s}-t-\frac{\left(x-x_{s}\right)}{g_{R}}\right)\right) \text { for }\left(x_{s}-g_{R} t\right) \leq\left(x-g_{R} t\right)<x_{s}, \\
\phi_{0}\left(x-g_{R} t\right) \text { for }\left(x-g_{R} t\right) \geq x_{s},
\end{array}\right.
$$

In Figure 4.4, we plot the density $\phi$ at time $t=0.2$. This corresponds to the time at which half the mass has already crossed the interface between zones $\Omega_{2}$ and $\Omega_{1}$. The solution is continuous when $g_{R}=2$, but exhibits a discontinuity in slope. We can draw the same conclusions as for the first test case. In particular, there are no oscillations in the neighborhood of the discontinuity, even if there is a discontinuity in the solution.

In panel b) of Figure 4.5 we plot in a logarithmic scale the $L 1$-norm error with respect to the space step $\Delta x$. The

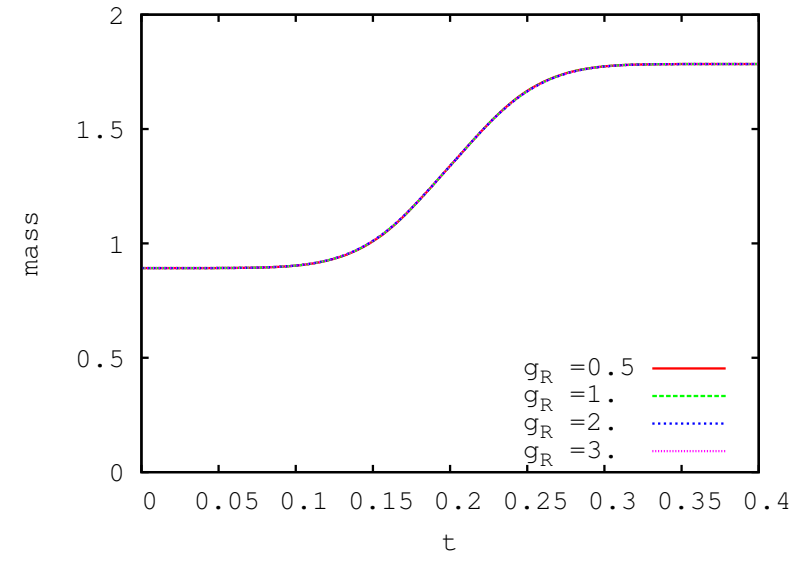

a) mass with respect to time

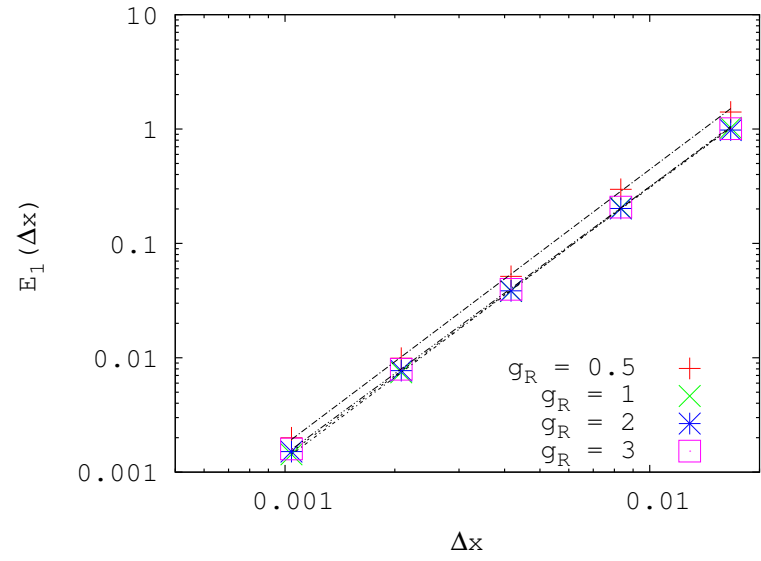

b) $L_{1}$-norm error with respect to $\Delta x$

FIGURE 4.5. $1 D$ test case 2. Doubling flux condition and velocity jump at interface $x=x_{s}$. The mass in panel a) has doubled when all the density has reached the second subdomain, as expected. In panel b) the $L_{1}$-norm error goes to zero with $\Delta x$. The convergence rates (around 2.4$)$ are displayed in Table 4.1.

convergence rates, gathered in Table 4.1, are similar to the first test case, which means that the transition condition has not reduced the precision of the scheme. We can notice that the lower the $g_{R}$, the worse the error. This is a similar situation to the first test case which can be explained by the same analogy with the traffic flow. In panel a) of Figure 4.5 we plot the mass with respect to time. Once all the cells have been transported in the right subdomain, the mass is doubled. As expected, since the speed is always $g_{L}=1$ in the left subdomain, in all cases the cells reach the interface at the same time and the mass profiles are identical. Figure 4.6 displays the 2D generalization of the second test case. 


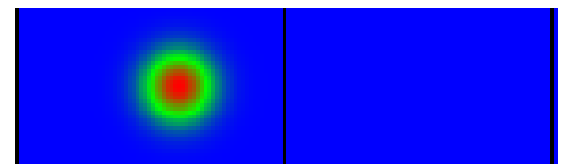

a) Initial time. The density bump is in zone $\Omega_{1}$.

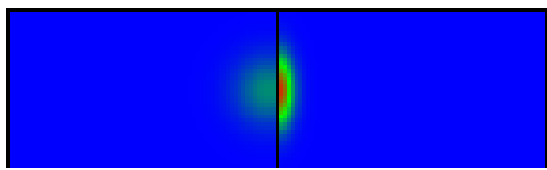

b) Intermediate time $t=0.16$.

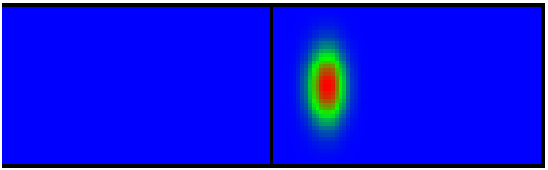

c) Final time $t=0.4$. The density

bump is in zone $\Omega_{2}$.

FIGURE 4.6. $2 D$ visualization of test case 2. Doubling flux condition and velocity jump at interface $x=x_{s}, u_{L}=1, u_{R}=0.5$. Snapshots of the density (initial time, passing interface, final time). The mass is doubling. (CFL $=0.4, \Delta x=0.001$ ).

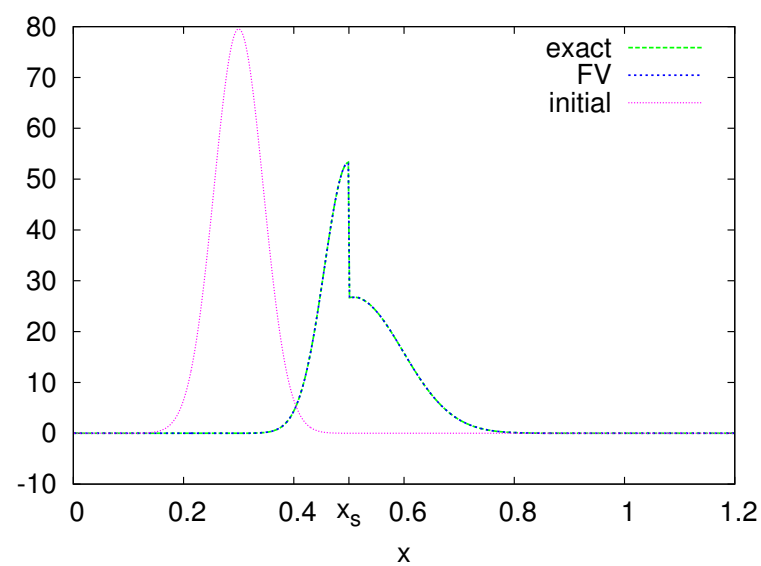

a) $g_{L}=0.5, t^{N}=0.4$

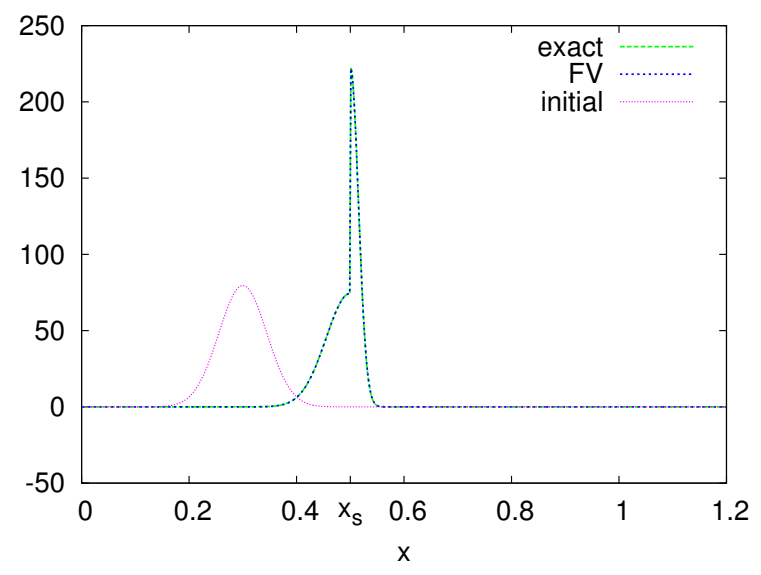

b) $g_{L}=3.0, t^{N}=0.0666$

FIGURE 4.7. $1 D$ test case 3: flux continuity condition and velocity jump at interface $x_{s}=0.5$, with source on the left. Snapshot of the density at initial time and when it crosses the interface between zones $\Omega_{1}$ and $\Omega_{2}\left(t=t^{N}\right)$.

Test case 3. Continuous flux, speed discontinuity on $\Omega_{1}-\Omega_{2}$ interface, linear source on the left. The third test case is the same as the first one except that we add a linear source term $\Lambda_{L}=1$ in the left part of the domain. The exact solution, detailed in Appendix, is

$$
\phi^{\text {exact }}(x, t)=\left\{\begin{array}{l}
\phi_{0}\left(x-g_{L} t\right) \exp (-t) \text { for } x<x_{s}, \\
g_{L} \phi_{0}\left(x_{s}-g_{L}\left(t-x-x_{s}\right)\right) \exp \left(-t+x-x_{s}\right) \text { for } x_{s}-t \leq x-t<x_{s}, \\
\phi_{0}(x-t) \text { for } x-t \geq x_{s},
\end{array}\right.
$$

As in the first test case, we plot the density at time $t^{N}$, when half the mass has already crossed the interface between zones $\Omega_{1}$ and $\Omega_{2}$. Two cases $g_{L}=0.5$ and $g_{L}=3$ are represented respectively on panels a) and b) of Figure 4.7. The convergence study is illustrated in panel b) of Figure 4.8. The scheme still works well with the source term, although the asymptotic order for the $L_{1}$-norm error is 2.4 , which is slightly less accurate than without the source term. Since the source term is active on the left subdomain, there is a loss in mass until the density bump reaches the interface (see panel a) of Figure 4.8). In the case where $g_{L}=3$, the mass stabilizes after the density has passed through the interface. In the case where $g_{L}=0.5$ the density bump has not entirely passed through the interface at $t=0.25$ so that the mass is not yet stabilized.

Test case 4. Doubling flux, speed discontinuity on $\Omega_{1}-\Omega_{2}$ interface, linear source. The fourth test case is the same as the second one except that we add a linear source term $\Lambda_{R}=1$ in the right part of the domain. The exact 
solution is then

$$
\phi^{\text {exact }}(x, t)=\left\{\begin{array}{l}
\phi_{0}(x-t) \quad \text { for } \quad x<x_{s}, \\
\frac{2}{g_{R}}\left(\phi_{0}\left(x_{s}-t-\frac{\left(x-x_{s}\right)}{g_{R}}\right) e^{\frac{x_{s}-x}{g_{R}}} \text { for }\left(x_{s}-g_{R} t\right) \leq\left(x-g_{R} t\right)<x_{s},\right. \\
\phi_{0}\left(x-g_{R} t\right) e^{-t} \text { for } \quad\left(x-g_{R} t\right) \geq x_{s},
\end{array}\right.
$$

As in the second test case, we plot the density at time $t=0.2$, for two values $g_{R}=0.5$ and $g_{R}=3$ respectively on panels a) and b) of Figure 4.9. Here also the asymptotic order of convergence for the error is roughly 2.4 (see panel b) of Figure 4.10). The source term is active in the right subdomain. The mass, observed in panel a) of Figure 4.10, first doubles as the density bump goes through the interface, then diminishes as soon as the loss term becomes active. The drop in mass begins at the same time in both cases $g_{R}=0.5$ and $g_{R}=3$ since the interface is reached with the same left speed.

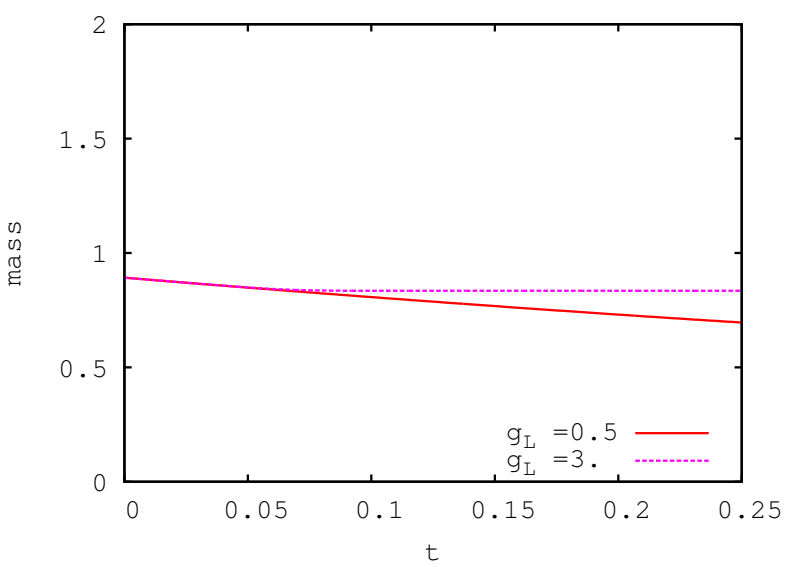

a) mass with respect to time

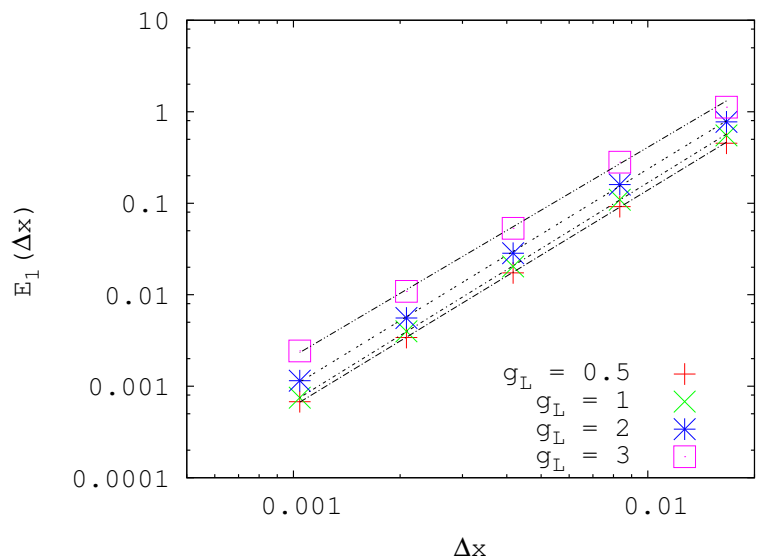

b) L1 norm error

FIGURE 4.8. $1 D$ test case 3: flux continuity condition and velocity jump at interface $x_{s}=0.5$, linear source on the left. In panel a) the mass decreases with time before the density has passed the interface ( $C F L=0.4, \Delta x=0.001)$. In panel $b)$ the $L_{1}$-norm error goes to zero with $\Delta x$. The convergence rates (around 2.4) are displayed in Table 4.1.

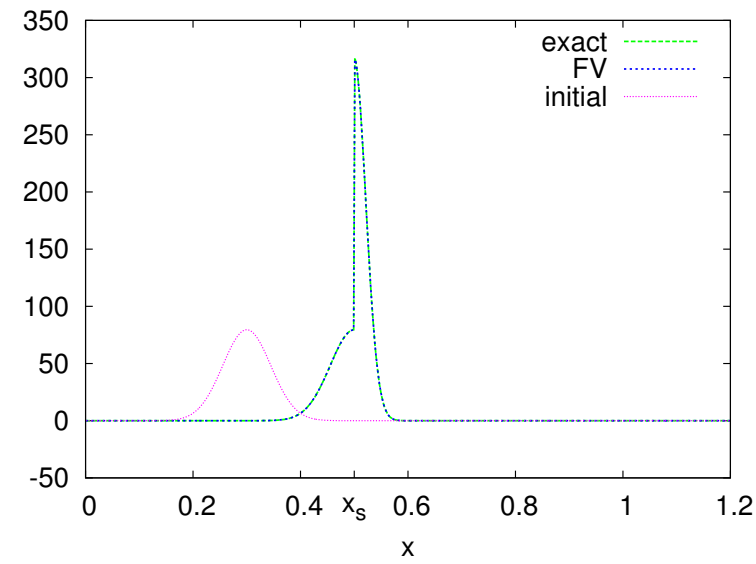

a) $g_{R}=0.5$

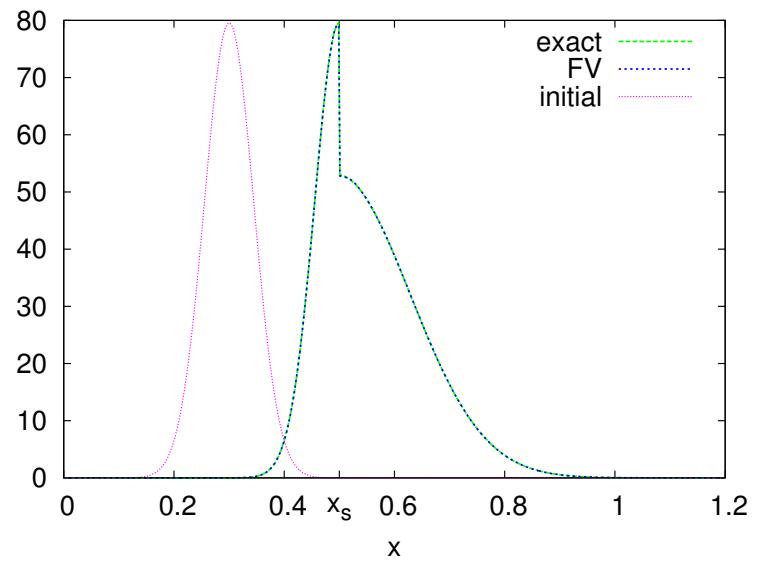

b) $g_{R}=3$

FIGURE 4.9. $1 D$ test case 4: doubling flux condition and velocity jump at interface $x_{s}=0.5$, linear source on the right. Snapshot of the density $\phi(x, t)$ at $t=0$ and $t=0.2$, when it crosses the interface between zones $\Omega_{1}$ and $\Omega_{2}$. 
4.2. 2D test cases. We now turn to the validation of the method in $2 \mathrm{D}$. As shown on Figure 2.1, the computing domain includes three internal boundaries: one vertical between zones $\Omega_{1}$ and $\Omega_{2}$, and two horizontal ones, between zones $\Omega_{1}$ and $\Omega_{3}$, and $\Omega_{2}$ and $\Omega_{3}$. The initial condition is centered on $\left(c_{x}, c_{y}\right)=(0.3,0.15)$.

Error Computing. In 2D the relative error due to discretization is computed with

$$
E(\Delta x)=\frac{\sum_{k=0}^{N x-1} \sum_{l=0}^{N y-1}\left|\phi_{k, l}^{N}-\bar{\phi}_{k, l}^{N}\right|}{\sum_{k=0}^{N x-1} \sum_{l=0}^{N y-1}\left|\bar{\phi}_{k, l}^{N}\right|}
$$

where $\bar{\phi}_{k, l}^{N}$ is the mean value of the exact solution on the grid mesh $\left[x_{k}, x_{k+1}\right] \times\left[y_{l}, y_{l+1}\right]$ at time $t^{N}$. This value is computed using the 2nd order quadrature formula.

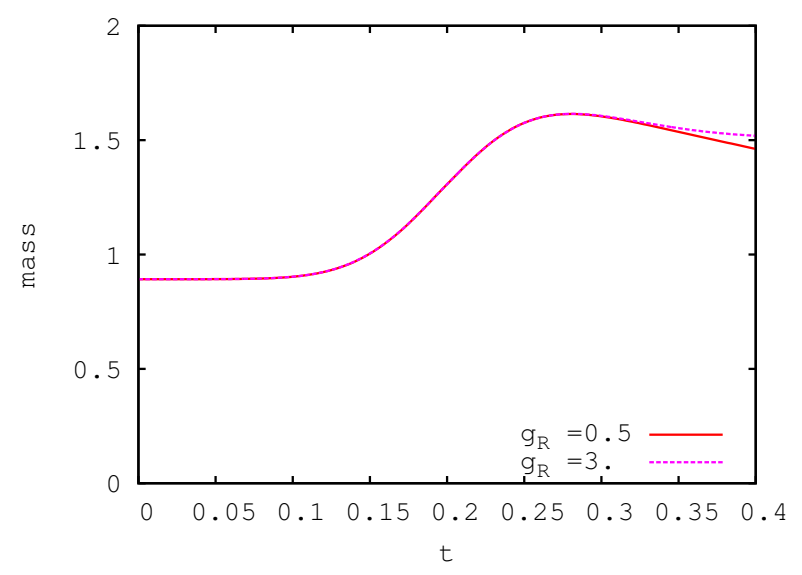

a) mass with respect to time

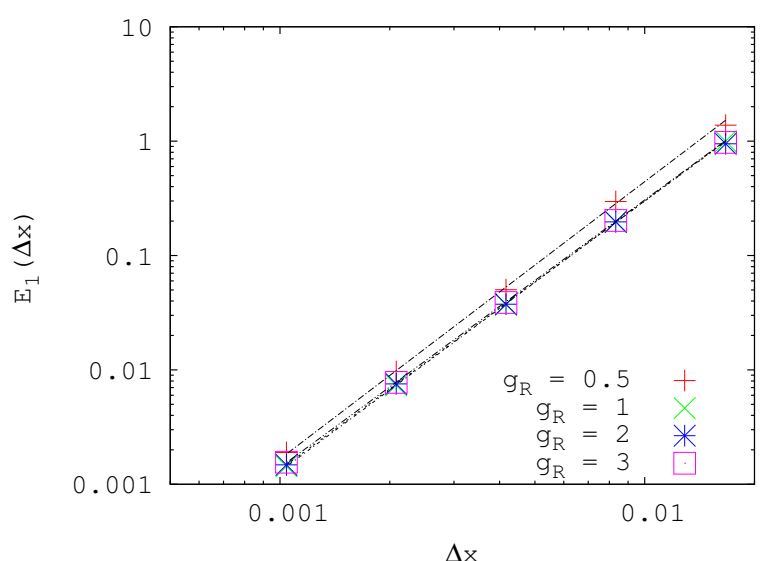

b) L1 norm error

FIGURE 4.10. $1 D$ test case 4: doubling flux condition and velocity jump at interface $x_{s}=0.5$, linear source on the right. The mass increases just after the interface, then decreases when the density has passed the interface. The convergence rates (around 2.4) are displayed in Table 4.1.

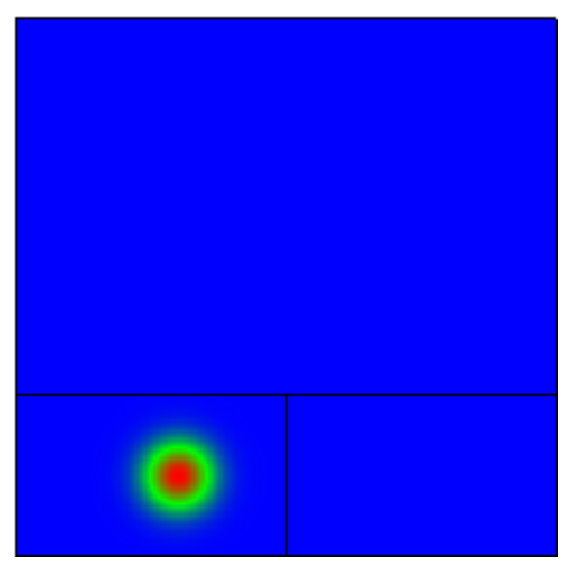

a) Initial time. The density bump lies in zone $\Omega_{1}$.

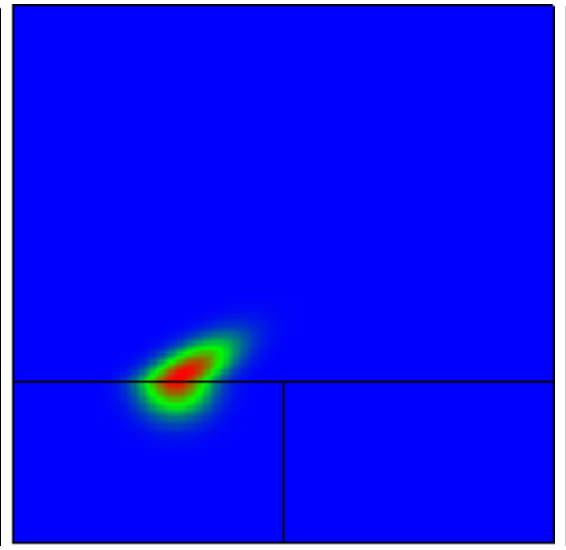

b) Intermediate time $t=0.15$.

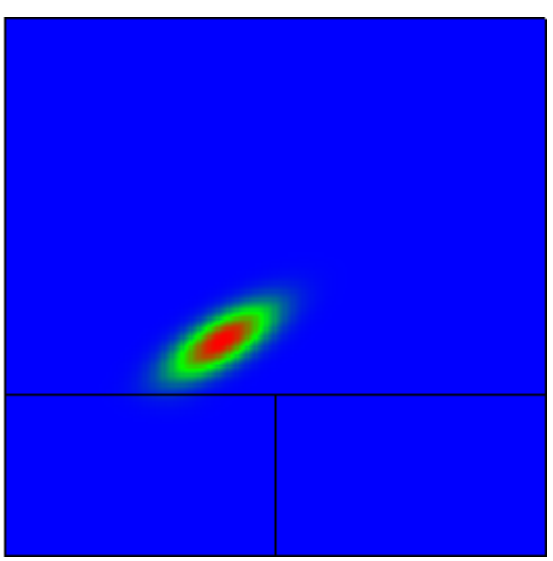

c) Final time $t=0.25$. The density bump lies in zone $\Omega_{3}$.

FIGURE 4.11. First $2 D$ test case, shear phenomenon. Snapshot of the density (initial time, passing interface, final time). $(C F L=0.4, \Delta x=$ 0.001). 
2D test case 1: shear interface. For this test case the speed is vertical $g(x, y)=0, h(x, y)=1$ in the bottom part of the computing domain $\left(\Omega_{1} \cup \Omega_{2}\right)$. In the top part $\left(\Omega_{3}\right)$, the speed is oblique $g(x, y)=1$ and $h(x, y)=1$. The discontinuity in speed is located on the horizontal internal boundary between $\Omega_{1}$ and $\Omega_{3}$ at $y_{s}=0.3$. On the snapshots in Figure 4.11, we can observe a shear phenomenon that could not occur in 1D. We also notice that the change of direction of the speed transforms the shape of the density bump, from circular to elliptic. The total mass remains constant. Due to the shear, the asymptotic rate of convergence of the error drops to 2.1 (see panel a) of Figure 4.12) .

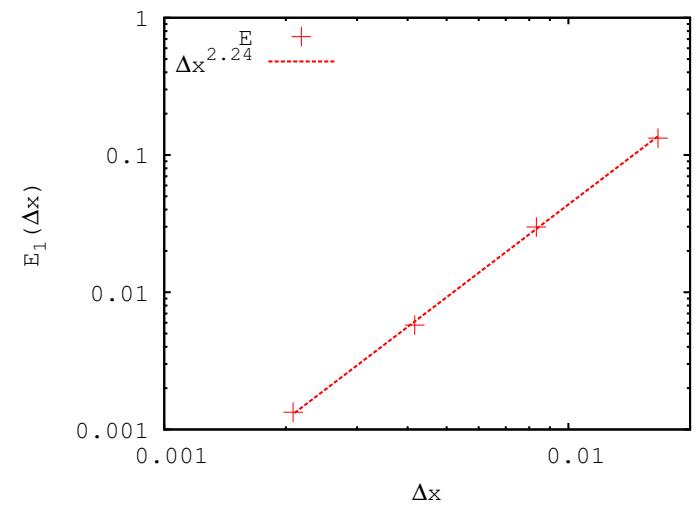

a) 2D test case 1: shear.

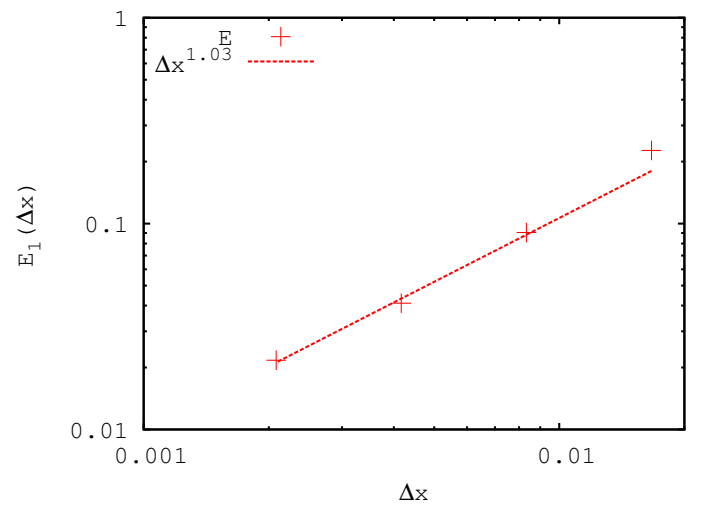

b) $2 D$ test case 2: waterproof.

FIGURE 4.12. $2 D$ test cases. $L_{1}$-norm relative error with respect to $\Delta x$. The convergence rate is 2.24 in the shear case (panel a) and drops to 1.03 in the waterproof case (panel $b$ ).

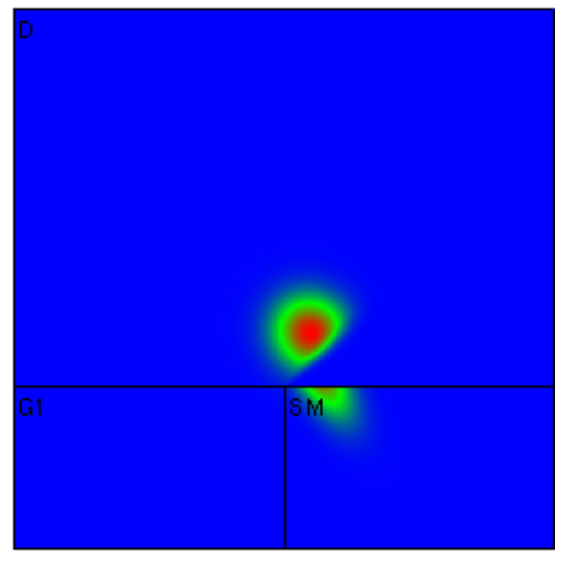

a) First order approximation.

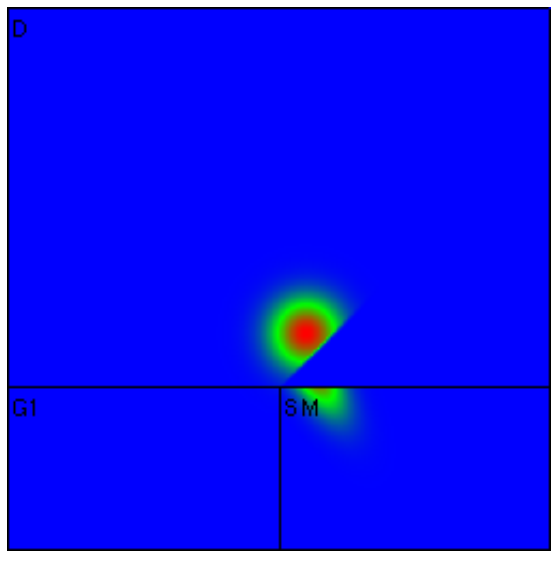

b) Third order approximation.

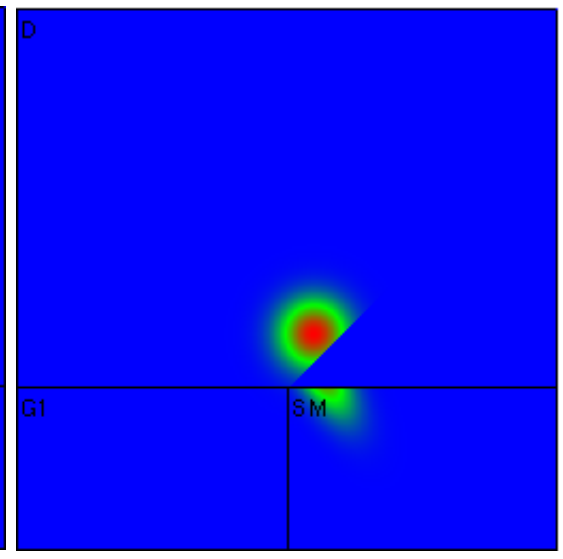

c) Exact solution.

FIGURE 4.13. 2D test case 2: waterproof interface. Density at final time (First and third order computations and exact solution). Some numerical diffusion affects the shape of the density, due to the splitting of the initial density bump into two separate clouds.

2D test case 2: waterproof interface. In this test case, the speed is diagonal $g(x, y)=1, h(x, y)=1$ in zone $\Omega_{1} \cup D$, and horizontal $g(x, y)=1, h(x, y)=0$ in zone $\Omega_{2}$. On the snapshots in Figure 4.13, we can observe a phenomenon of waterproof interface that could not occur in 1D. The fraction of the mass which crosses the vertical interface between zones $\Omega_{1}$ and $\Omega_{2}$ remains trapped in zone $\Omega_{2}$ and can only move horizontally. The convergence rate drops to 1 (panel b) of Figure 4.12). In order to understand better the drop in precision for the 2D test case 2, we have studied other values for $h(x, y)=h_{B}$ in zone $\Omega_{2}$, ranging from the reference case with no speed variation $\left(h_{B}=1\right)$ to the waterproof test case $\left(h_{B}=0\right)$. Figure 4.14 displays the density at time $t=0.25$ for three different $h_{B}$ values. For $h_{B}=0.9$, the solution displayed on panel a) looks very much like that obtained with the reference case. For $h_{B}=0.001$, the solution displayed in panel c) looks very much like that obtained with the waterproof boundary in 
Figure 4.13.

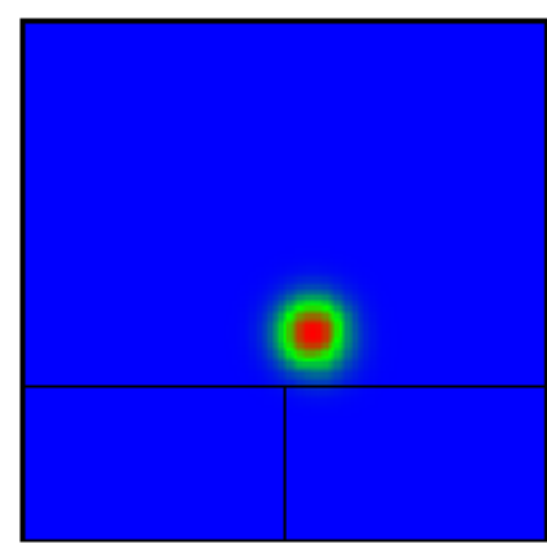

a) $h_{B}=0.9$

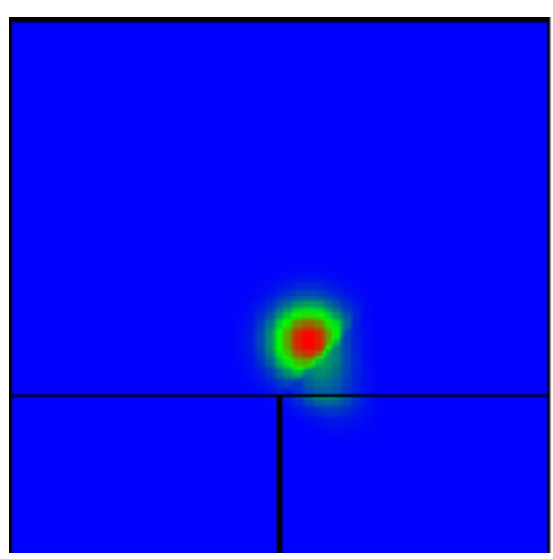

b) $h_{B}=0.5$

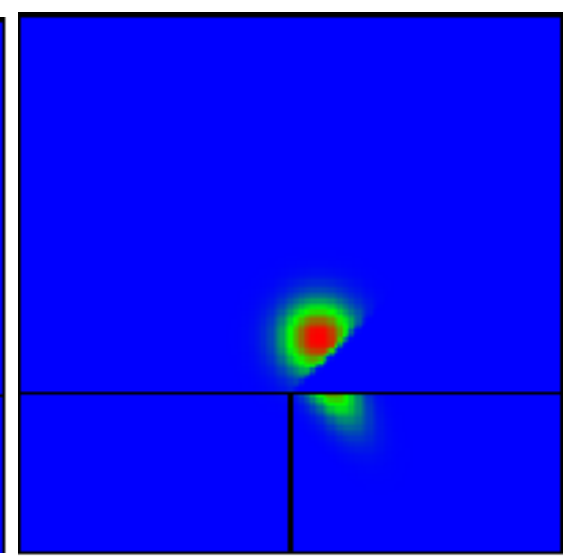

c) $h_{B}=0.001$

FIGURE 4.14. From constant diagonal speed to waterproof interface. Solution at time $t=0.25$ for three different values of the vertical speed in the $\Omega_{2}$ zone. a) $\left.\left.h_{B}=0.9, b\right) h_{B}=0.5, c\right) h_{B}=0.001$

Since the exact solution is not available except for the extreme values $h_{B}=0$ or 1 , we compute the error at time $t^{N}$ using in (4.3), instead of the exact solution, a reference solution $\phi^{\text {fine }}$ computed on a very fine grid with $N_{x}^{\text {fine }}$ grid meshes, and projected on the current grid with $N_{x}^{\text {coarse }}$ grid meshes:

$$
\bar{\phi}_{k, l}^{N}=\sum_{i=1}^{m} \sum_{j=1}^{m} \phi_{k m+i, l m+j}^{f i n e, N}
$$

with

$$
m=\frac{N_{x}^{\text {fine }}}{N_{x}^{\text {coarse }}}
$$

Here the space step for the reference solution is $\Delta x=0.0005$, corresponding to $N_{x}^{f i n e}=2000$. The different error curves and the variation of the error order with respect to $h_{B}$ are displayed respectively on panel a) and b) of Figure 4.15. This numerical experiment confirms that there is a regular drop in precision as the contrast between the vertical speeds on both sides of the interface between zones $\Omega_{2}$ and $\Omega_{3}$ increases. It is worth noting that the asymptotic order for the waterproof case $\left(h_{B}=0\right)$ computed using the converged solution is 1.25 instead of the value 1 obtained when using the exact solution in formula (4.3). On the other hand, the asymptotic order for the case with constant diagonal speed $\left(h_{B}=1\right)$ is 2.25 , which is less than the asymptotic order 2.4 obtained in the $1 \mathrm{D}$ test case 1 for $g_{L}=g_{R}=1$. This small drop in precision is generally encountered with $2 \mathrm{D}$ schemes on cartesian grids obtained by tensorization of a 1D scheme.

5. Application to cell dynamics. In this last section we turn to the numerical simulation of a biological model dedicated to the cell dynamics in ovarian follicular development described in Echenim et al. [13] and references therein. This multiscale model describes the latest stages of the follicular development before ovulation. The cell population of each follicle is studied as a density function depending on time, and structured according to two functional space variables, age and maturity. The density functions of the interacting follicles are solutions of weakly coupled PDE$\mathrm{s}$, where the age and maturity velocities and the loss term depend on the integro-moments of the solution for all the follicles. The moment-based integral formulation accounts in a compact way for the feedback loop involving the ovarian hormones, secreted from follicular cells, and the pituitary hormone FSH (follicle-stimulating hormone) that targets in turn the follicular cells.

The model follows the development of the follicles starting from an initial stage where all cells are proliferating. As the granulosa cells progress through subsequent cell cycles their maturities increase up to a threshold beyond which they 


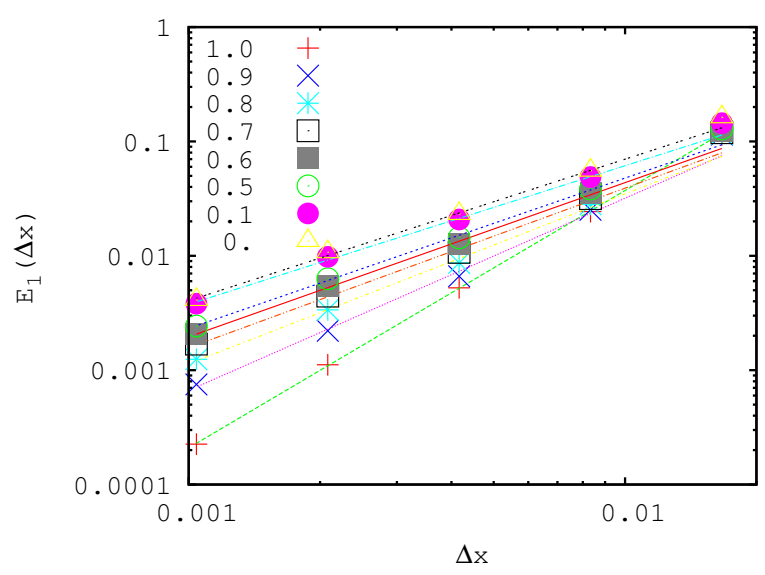

a) Relative error variation.

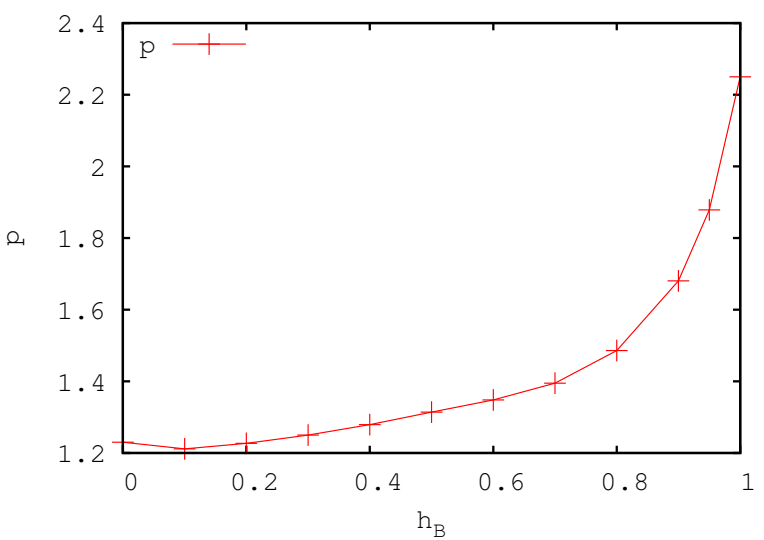

b) Order p as function of $h_{B}$.

FIGURE 4.15. From constant diagonal speed to waterproof interface. a) Relative error and best least-square fit $O\left(\Delta x^{p}\right)$ for different constant $h_{B}$ values of the vertical speed in zone $\Omega_{2}$. b) Asymptotic order $p$ as a function of $h_{B}$ value in zone $\Omega_{2}$.

exit the proliferation cell cycle and enter the differentiation stage D. The control exerted by FSH on the cells depends on the cell state, that is on whether the cell be within or outside the division cycle. Moreover, cells become insensitive to any external control during a part of the cycle, leading to a drift dynamics behaving as pure transport.

Cell proliferation is underlaid by the process of mitosis, through which a mother cell gives birth to two daughter cells. Mitosis is the endpoint of the cell cycle that consists of the 4 phases G1, S, G2 and M, and ensures proper DNA replication and repartition between the new cells. The most common way of representing mitosis in age-structured models of cell populations is to add a gain term in the right hand side, that controls the (average) doubling time in the cell population (see the renewal equations presented in Chapter 3 of [18]). This is however a rough description of the mitosis event since it is distributed over all cell ages. An alternative way to represent mitosis is to consider appropriate boundary conditions coupling the dynamics of the population of proliferating cells with another population of cells that have exited the cell cycles. Instances of corresponding models can be found in the context of hematopoiesis, the process by which blood cells are produced (see e.g. [2]).

Here, we consider a model where one cannot get rid of discontinuity problems, since not only the mitosis event, but also the distinction between different phases of the cell cycle are embedded in the cell population dynamics. Due to the phasedependent sensitivity of cells to the extracellular signals that make them progress along the cell cycle, discontinuities on the velocities have to be dealt with in addition to the mitosis-induced discontinuity. More precisely, we account for both the START transition from phase G1 to S and the ExIT transition after mitosis completion [22]. Besides the mitosis process which increases the total cell mass, the follicular development model takes into account apoptosis, and therefore total mass loss, via a source term which is active only locally in a narrow zone delimited by a skewed gaussian law and centered on the boundary between the first phase G1 of the cycle and the differentiation phase. The coefficients of the cell loss term depend on the first moment of the solution.

An additional difficulty compared with the toy models is the weak coupling between different follicles through the generalized moments of the cell density. The HPC issues in the case of realistic numbers of follicles -around 20- have already been addressed in [5]. Here we will first focus on the case of one follicle to study the precision of the scheme, and then illustrate the vector case with a competition between two follicles. Let us denote by $F$ the number of follicles in the general case. The density $\Phi=\left(\phi_{f}\right)_{f=1, \ldots, F}$ satisfies the following system of equations, for $f=1, \ldots, F$ :

$$
\frac{\partial \phi_{f}(x, y, t)}{\partial t}+\frac{\partial\left(g_{f}\left(x, y, u_{f}(t)\right) \phi_{f}(x, y, t)\right)}{\partial x}+\frac{\partial\left(h_{f}\left(x, y, u_{f}(t)\right) \phi_{f}(x, y, t)\right)}{\partial y}=-\Lambda(x, y, U(t)) \phi_{f}(x, y, t)
$$

set in the computing domain $\Omega$ in the $(x, y)$ plane,

$$
\Omega=\left\{(x, y), 0 \leq x \leq N_{c} \times D, 0 \leq y \leq 1\right\}
$$




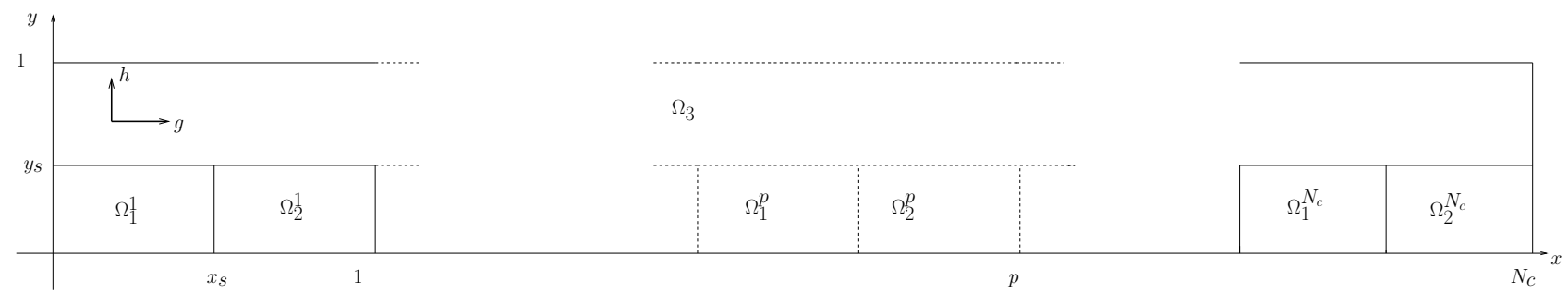

a) Computational domain. The cell cycles $\Omega_{1}^{p} \cup \Omega_{2}^{p}, p=1, \ldots, N_{c}$ of unit width are periodically reproduced.

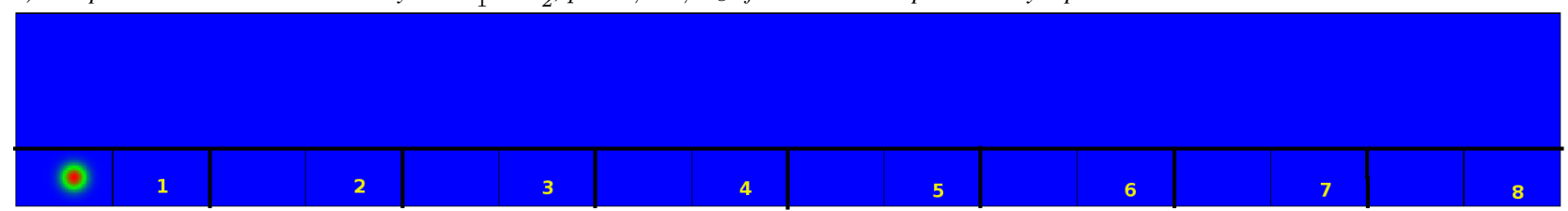

b) Initial condition, identical for both follicles. The simulation covers $N_{c}=8$ cell cycles. Each cell cycle is divided into $\Omega_{1}^{p} \cup \Omega_{2}^{p}$, for $p=1, \ldots, 8$. The cell population is initially in $\Omega_{1}^{1}$, in the first cell cycle. A color code is used for the cell density; a null density is encoded in blue, while the highest value is encoded in red. $\phi_{\max }=75$ denotes the maximum observed density over the whole domain at $t=0$.

FIGURE 5.1. Initial condition and computational domain for the full model

where $N_{c}$ is the number of cell cycles and $D$ is the duration of one cycle. The domain $\Omega$ is divided in $\mathcal{N}=2 N_{c}+1$ zones: $\Omega_{1}^{p}, \Omega_{2}^{p}$, for $p=1, \ldots, N_{c}$ and $\Omega_{3}$, corresponding to different cell states illustrated in panel a) of Figure 5.1 and hence different definition of the speeds and source terms. Phase $\Omega_{2}^{p}$ in the model aggregates the three latest phases $(\mathrm{S}$, $\mathrm{G} 2, \mathrm{M})$ of the $p^{t h}$ cell cycle

$$
\left\{\begin{array}{l}
\Omega_{1}^{p}=\left\{(x, y) \in \Omega, \quad p D \leq x \leq(p+1 / 2) D, \quad 0 \leq y \leq y_{s}\right\}, \quad p=0, \ldots, N_{c}-1, \quad \Theta_{1}=\sum_{p=1}^{N_{c}} \Omega_{1}^{p} \\
\Omega_{2}^{p}=\left\{(x, y) \in \Omega, \quad(p+1 / 2) D \leq x \leq(p+1) D, \quad 0 \leq y \leq y_{s}\right\}, \quad p=0, \ldots, N_{c}-1, \quad \Theta_{2}=\sum_{p=1}^{N_{c}} \Omega_{2}^{p} \\
\Omega_{3}=\left\{(x, y) \in \Omega, \quad y_{s} \leq y\right\} .
\end{array}\right.
$$

Each cell cycle consists of the $\Omega_{1}^{p} \cup \Omega_{2}^{p}$ subdomain and $\Theta_{i}$ for $i=1,2$ denotes the disconnected union of the $N_{c}$ corresponding phases $\Omega_{p}^{i}$, for $p=1, \ldots, N_{c}$.

The aging function $g_{f}$ appearing in (5.1) is defined by

$$
g_{f}(x, y, u)= \begin{cases}\gamma_{1} u+\gamma_{2} & \text { for }(x, y) \in \Theta_{1} \\ 1 & \text { for }(x, y) \in \Theta_{2} \cup \Omega_{3}\end{cases}
$$

where $\gamma_{1}, \gamma_{2}$ are real positive constants that may depend on the follicle $f$.

The maturation function $h_{f}$ is defined by

$$
h_{f}(x, y, u)= \begin{cases}\tau_{h}\left(-y^{2}+\left(c_{1} y+c_{2}\right)\left(1-\exp \left(\frac{-u}{\bar{u}}\right)\right)\right) & \text { for }(x, y) \in \Theta_{1} \cup \Omega_{3} \\ 0 & \text { for }(x, y) \in \Theta_{2}\end{cases}
$$

where $\tau_{h}, c_{1}, c_{2}$ and $\bar{u}$ are real positive constants that may depend on the follicle $f$.

The source term, that represents cell loss through apoptosis, is defined by

$$
\Lambda(x, y, U)= \begin{cases}\bar{\Lambda} \exp \left(-\left(\frac{\left(y-y_{s}\right)^{2}}{\bar{\gamma}}\right)\right) \times(1-U) & \text { for }(x, y) \in \Theta_{1} \cup \Omega_{3} \\ 0 & \text { for }(x, y) \in \Theta_{2}\end{cases}
$$

where $\bar{\Lambda}, y_{s}$ and $\bar{\gamma}$ are real positive constants.

The equations in the PDE system (5.1) are linked together through the argument $u_{f}(t)$ appearing in the speeds $g_{f}(x, y, u)$ 


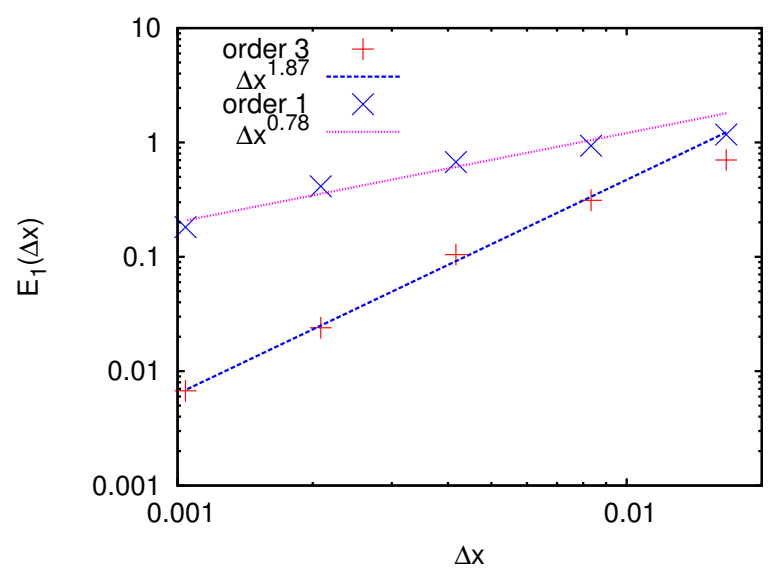

FIGURE 5.2. Follicular development model. Symbols $\times$ and + indicate the numerical errors respectively obtained with the order 1 and order 3 schemes. Dotted lines indicate the corresponding numerical convergence rates (0.78 and 1.87) obtained by least-square fit.

and $h_{f}(x, y, u)$ and the argument $U(t)$ appearing in the source term $\Lambda(x, y, U) . U(t)$ and $u_{f}(t)$ represent respectively the plasma FSH level and the locally bioavailable FSH level and depend on the first maturity moment of the densities

$$
\begin{gathered}
m_{f}(t)=\int_{0}^{1} \int_{0}^{N_{c} D} y \phi_{f}(x, y, t) \mathrm{d} x \mathrm{~d} y . \\
M(t)=\sum_{f=1}^{F} m_{f}(t)
\end{gathered}
$$

Note that in the case of one single follicle $m_{f}(t)$ and $M(t)$ are identical.

The plasma FSH level $U(t)$ showing up in the arguments of the source term in (5.1) is defined by

$$
U(t)=U_{\min }+\frac{1-U_{\min }}{1+\exp (c(M(t)-\bar{M}))},
$$

where $U_{\min }, c$ and $\bar{M}$ are real positive constants.

The locally bioavailable FSH level $u_{f}(t)$ showing up in the arguments of the speeds in (5.1) is defined by

$$
u_{f}(t)=\min \left(b_{1}+\frac{e^{b_{2} m_{f}(t)}}{b_{3}}, 1\right) U(t), \quad \text { for } f=1, \ldots, F
$$

where $b_{1}, b_{2}$ and $b_{3}$ are real positive constants that may depend on the follicle $f$. The numerical value of the biological constants appearing in definitions 5.2, 5.3 and 5.8 are gathered in Table 4.1. To simplify the notation we do not explicitly indicate their possible dependence as a function of the follicle even if it is implied by the $f$ index notation.

The precise definition of the required transmission conditions along the successive cell cycles of the domain has been formulated in [20]. For each cycle $p=1, \ldots, N_{c}$,

- the flux on the $x$-axis is continuous on the interface between $\Omega_{1}^{p}$ and $\Omega_{2}^{p}$

$$
\phi_{f}\left(t, x^{+}, y\right)=\left(\gamma_{1} u_{f}+\gamma_{2}\right) \phi_{f}\left(t, x^{-}, y\right), \quad x=(p-1 / 2) D, \quad 0 \leq y \leq y_{s} .
$$

- The flux is doubling on the interface between $\Omega_{2}^{p}$ and $\Omega_{1}^{p+1}$, which accounts for the birth of two daughter cells from one mother cell at the end of each cell cycle

$$
\left(\gamma_{1} u_{f}+\gamma_{2}\right) \phi_{f}\left(t, x^{+}, y\right)=2 \phi_{f}\left(t, x^{-}, y\right), \quad x=p D, \quad 0 \leq y \leq y_{s}
$$


- A homogeneous Dirichlet condition holds to the north of the interface between $\Omega_{2}^{p}$ and $\Omega_{3}$

$$
\phi_{f}\left(t, x, y_{s}^{+}\right)=0, \quad(p-1 / 2) D \leq x \leq p D .
$$

We refer the reader to [5] and the references herein for more details on the model, but nevertheless stress out that, as mentioned in the introduction, an important feature, which is inherent to cell dynamics, is the mitosis event occurring at the end of each cell cycle, materialized in our setup by the interfaces between phases $\Omega_{2}^{p}$ and $\Omega_{1}^{p+1}$. In most transport equations dedicated to cell dynamics, it is not accounted for as such but rather by a gain term distributed over the cell population [18]. In [20], the model with discontinuous coefficients was shown to have a unique weak solution, provided that the discontinuities in the velocities were taken into account by flux continuity - at the interfaces between phases $\Omega_{1}^{p}$ and $\Omega_{2}^{p}$ - and flux doubling - at the end of each cycle. The $\Omega_{2}$ phase being a transport in age direction only, null flux at the interface with the differentiating phase $\Omega_{3}$ ensures the well posedness.

5.1. Numerical simulation. We first perform a convergence study on the full model. For this time consuming validation, we restrict ourselves to only one follicle $F=1$, and we stop the simulation after a duration $t^{N}=1$, which is short enough so that the computations on very fine grids remain tractable and would correspond to the transit time across the first cell cycle if there were no control of the ageing velocity (i.e. if the cell age evolved as time in $\Omega_{1}^{1}$ ). Even if, for the chosen values of the biological parameters, gathered in Table 5.1, the first cell cycle duration is slightly less than one, all of the three interesting transitions: $\Omega_{1}^{1}-\Omega_{2}^{1}, \Omega_{2}^{1}-\Omega_{1}^{2}$ and $\Omega_{1}^{2}-\Omega_{3}$ do happen, as well as the waterproof phenomenon between zones $\Omega_{2}^{1}-\Omega_{3}$. The relative error is computed with respect to a converged solution obtained using a very fine grid of 1920 grid meshes per half cycle (see equation 4.4). It is displayed as a function of the space step $\Delta x$ on Figure 5.2. The asymptotic order of convergence computed by least square fitting is almost 2 . The biological model combines all the numerical difficulties studied separately in the six previous test cases. As expected, the order of convergence is intermediate between the best and worst order achieved for these toy problems. Finally we illustrate the competition between two follicles. The initial condition, with all cells in phase $\Omega_{1}^{1}$, is represented in the bottom panel of Figure 5.1. To illustrate the different phenomena at interfaces, snapshots of the density at significant times of the follicular development are displayed on Figures 5.3 and 5.4. For this simulation, a space discretization $\Delta x=0.0125$ ( 80 grid meshes per half cycle) is used, leading to a varying time discretization of $\Delta t \approx 0.0012$ which meets condition (3.9) with $C F L=0.4$. The snapshots illustrate two important aspects of the model. First, the transitions between adjacent phases are well captured using the numerical method described in the paper. Second, the differences between two competing follicles whose biological properties differ only by the value of one parameter, the maturation velocity, are shown to increase with time. At initial time the two follicles have the same cell population $m_{0}^{1}=m_{0}^{2}=1$, and in the final snapshot at $t=6.30$ (panel b) of Figure 5.4), which corresponds to a proliferation process covering seven cell cycles, their cell populations are very different in term of cell mass and cell repartition within the domain. Follicle 1 (top), which is $70 \%$ slower in the maturity axis (y) than follicle 2 (bottom), is still evolving towards the asymptotic maturity level and its density is still split into distinct clouds corresponding to cells having entered the differentiation phase during different cell cycles, with a total mass $m_{0}^{1}=9.3$. At the same time, follicle 2 has almost reached its asymptotic state with a final total mass of only $m_{0}^{2}=4.3$. The difference in mass can be explained by the fact that being slower in the $y$ direction, follicle 1 spends more time in the proliferation phase than follicle 2 .

Conclusion. The numerical method presented in Sections 2 and 3 and validated by the numerical convergence study in Section 4 is applied to a biological model for follicular development. Thanks to the original treatment of interface conditions it shows very promising results in terms of accuracy. This numerical tool will enable us to calibrate the model with respect to quantitative biological specifications. More extensive simulations with realistic numbers of follicles in competition will be performed to better understand the phenomena under study, like for instance multiple ovulations.

\section{REFERENCES}

[1] Adimurthi, S. Mishra, and G. D. V. Gowda. Optimal entropy solutions for conservation laws with discontinuous flux-functions. J. Hyperbolic Differ. Equ., 2(4):783-837, 2005. 
B. Aymard, F. Clément, F. Coquel and M. Postel

\begin{tabular}{|c|c|c|c|}
\hline Parameter & Description & \multicolumn{2}{|c|}{ Value } \\
\hline $\begin{array}{l}U_{\min } \\
\frac{c}{M}\end{array}$ & $\begin{array}{l}\text { FSH plasma level (eq. (5.7)) } \\
\text { minimum level } \\
\text { slope parameter } \\
\text { abscissa of the inflection point }\end{array}$ & \multicolumn{2}{|c|}{0.5} \\
\hline $\begin{array}{l}\bar{\Lambda} \\
\bar{\gamma}\end{array}$ & $\begin{array}{l}\text { Apoptosis source term (eq. (5.4)) } \\
\text { intensity factor } \\
\text { scaling factor }\end{array}$ & \multicolumn{2}{|c|}{$\begin{array}{c}0.1 \\
0.01\end{array}$} \\
\hline$y_{s}$ & cellular maturity threshold & \multicolumn{2}{|c|}{0.3} \\
\hline $\begin{array}{l}b_{1} \\
b_{2} \\
b_{3}\end{array}$ & $\begin{array}{c}\text { intrafollicular FSH level (eq. (5.8)) } \\
\text { basal level } \\
\text { exponential rate } \\
\text { scaling factor }\end{array}$ & \multicolumn{2}{|c|}{$\begin{array}{r}0.08 \\
2.25 \\
1450 .\end{array}$} \\
\hline $\begin{array}{l}\gamma_{1} \\
\gamma_{2}\end{array}$ & $\begin{array}{l}\text { Aging function (eq. (5.2)) } \\
\text { rate } \\
\text { origin }\end{array}$ & \multicolumn{2}{|c|}{$\begin{array}{l}2 . \\
2 .\end{array}$} \\
\hline $\begin{array}{c}c_{1} \\
c_{2} \\
\bar{u}\end{array}$ & \multirow[t]{2}{*}{ Maturation function (eq. (5.3)) } & \multicolumn{2}{|c|}{$\begin{array}{l}0.68 \\
0.08 \\
0.02\end{array}$} \\
\hline$\tau_{h}$ & & $\begin{array}{c}\text { foll. } 1 \\
0.7\end{array}$ & $\begin{array}{c}\text { foll. } 2 \\
1.0\end{array}$ \\
\hline
\end{tabular}

TABLE 5.1

Values of the parameters for the biological model simulation. The two follicles in competition differ by the value of $\tau_{h}$

[2] M. Adimy, F. Crauste, and S. Ruan. A mathematical study of the hematopoiesis process with applications to chronic myelogenous leukemia. SIAM J. Appl. Math., 65(4):1328-1352, 2005.

[3] A. Ambroso, C. Chalons, F. Coquel, E. Godlewski, F. Lagoutière, P.-A. Raviart, and N. Seguin. Relaxation methods and coupling procedures. Internat. J. Numer. Methods Fluids, 56(8):1123-1129, 2008.

[4] E. Audusse and B. Perthame. Uniqueness for scalar conservation laws with discontinuous flux via adapted entropies. Proc. Roy. Soc. Edinburgh Sect. A, 135(2):253-265, 2005.

[5] B. Aymard, F. Clément, F. Coquel, and M. Postel. Numerical simulation of the selection process of the ovarian follicles. To appear in ESAIM Proc., http://hal.archives-ouvertes.fr/hal-00656382, 2012.

[6] F. Bouchut and F. James. One-dimensional transport equations with discontinuous coefficients. Nonlinear Anal., 32(7):891-933, 1998.

[7] B. Boutin, F. Coquel, and L. P. G. Coupling techniques for nonlinear hyperbolic equations. iii. well-balanced approximation of thick interfaces. arXiv:1205.2437, 2012.

[8] R. Bürger and K. H. Karlsen. Conservation laws with discontinuous flux: a short introduction. J. Engrg. Math., 60(3-4):241-247, 2008.

[9] R. Bürger, K. H. Karlsen, and N. H. Risebro. A relaxation scheme for continuous sedimentation in ideal clarifier-thickener units. Comput. Math. Appl., 50(7):993-1009, 2005.

[10] R. Bürger, K. H. Karlsen, and J. D. Towers. A conservation law with discontinuous flux modelling traffic flow with abruptly changing road surface conditions. In Hyperbolic problems: theory, numerics and applications, volume 67 of Proc. Sympos. Appl. Math., pages $455-464$. Amer. Math. Soc., Providence, RI, 2009.

[11] G.-Q. Chen, N. Even, and C. Klingenberg. Hyperbolic conservation laws with discontinuous fluxes and hydrodynamic limit for particle systems. J. Differ. Equations, 245(11):3095 - 3126, 2008.

[12] N. Echenim. Modélisation et contrôle multi-échelles du processus de sélection des follicules ovulatoires. PhD thesis, Université Paris-Sud XI, Faculté des Sciences d'Orsay, 2006.

[13] N. Echenim, D. Monniaux, M. Sorine, and F. Clément. Multi-scale modeling of the follicle selection process in the ovary. Math. Biosci., 198(1):57-79, 2005.

[14] E. Godlewski and P.-A. Raviart. The numerical interface coupling of nonlinear hyperbolic systems of conservation laws. I. The scalar case. Numer. Math., 97(1):81-130, 2004.

[15] S. Gottlieb and C. W. Shu. Total variation diminishing runge kutta schemes. Math. Comp., 67(221):73-85, 1998.

[16] B. Koren. A robust upwind discretisation method for advection, diffusion and source terms. In Numerical Methods for Advection-Diffusion Problems, page 117. Braunschweig: Vieweg, 1993.

[17] S. N. Kružkov. First order quasilinear equations with several independent variables. Mat. Sb. (N.S.), 81 (123):228-255, 1970.

[18] B. Perthame. Transport Equations in Biology. Birkhaüser Verlag, Basel, 2007.

[19] N. Seguin and J. Vovelle. Analysis and approximation of a scalar conservation law with a flux function with discontinuous coefficients. Math. Models Methods Appl. Sci., 13(02):221-257, 2003.

[20] P. Shang. Cauchy problem for multiscale conservation laws : Applications to structured cell populations. http://arxiv.org/abs/1010.2132, 2010. 
[21] P. K. Sweby. High resolution schemes using flux limiters for hyperbolic conservation laws. SIAM J. Numer. Anal., 21(5):995-1011, 1984.

[22] J. J. Tyson and B. Novak. Temporal organization of cell cycle. Curr. Biol., 18:R759-768, 2008.

Appendix A. Exact solutions. We now give the details of the exact solution computations for the 1D and 2D test cases.

\section{A.1. Exact solutions of the 1D problem with piecewise constant speeds.}

\section{Problem.}

$$
\left\{\begin{array}{l}
\partial_{t} \phi+\partial_{x} g \phi=\Lambda \phi \quad \text { for } \quad\left[0, x_{s}\right] \cup\left[x_{s}, x_{L}\right] \\
g(x)=\left\{\begin{array}{lll}
g_{L} & \text { if } \quad x<x_{s} & \text { (discontinuous speed) } \\
g_{R} & \text { if } & x \geq x_{s}
\end{array}\right. \\
\Lambda(x)=\left\{\begin{array}{lll}
\Lambda_{L} & \text { if } \quad x<x_{s} \\
\Lambda_{R} & \text { if } & x \geq x_{s}
\end{array}\right. \\
\phi(x, 0)=\phi_{0}(x)
\end{array}\right.
$$

Case without source term. We first solve this problem when $\Lambda_{L}=\Lambda_{R}=0$, using the method of characteristics. The case when $g_{R}<g_{L}$ is displayed in Figure A.1. If there were no transmission conditions, the characteristics would be straight lines passing through the vertical axis. The analysis is detailed in the case where $g_{L}$ and $g_{R}$ speeds are positive which is the most usual situation in problems arising from biology.

Solution. For $x<x_{s}$, considering the characteristics of

$$
\left\{\begin{array}{l}
\partial_{t} \phi+g_{L} \partial_{x} \phi=0 \\
\phi(x, 0)=\phi_{0}(x)
\end{array}\right.
$$

the solution is

$$
\phi(x, t)=\phi_{0}\left(x-g_{L} t\right) \text { for } x \leq x_{s} .
$$

Using the same argument, considering the characteristics of

$$
\left\{\begin{array}{l}
\partial_{t} \phi+g_{R} \partial_{x} \phi=0, \\
\phi(x, 0)=\phi_{0}(x),
\end{array}\right.
$$

we find that

$$
\phi(x, t)=\phi_{0}\left(x-g_{R} t\right) \quad \text { for } \quad\left(x-g_{R} t\right) \geq x_{s} .
$$

The solution for $\left(x_{0}-g_{R} t\right)<\left(x-g_{R} t\right) \leq x_{s}$ is obtained thanks to the transmission condition

$$
\psi_{L}\left(g_{L} \phi\left(x_{s}^{-}, t\right)\right)=g_{R} \phi\left(x_{s}^{+}, t\right),
$$

combined with the fact that

$$
\phi\left(x_{s}^{-}, t\right)=\phi_{0}\left(x_{s}-g_{L} t\right),
$$

which leads to

$$
g_{R} \phi\left(x_{s}^{+}, t\right)=\psi_{L}\left(g_{L} \phi\left(x_{s}^{-}, t\right)\right)=\psi_{L}\left(g_{L} \phi_{0}\left(x_{s}-g_{L} t\right)\right) .
$$


Defining a trace function

$$
\operatorname{Tr}(t)=\frac{1}{g_{R}} \psi_{L}\left(g_{L} \phi_{0}\left(x_{s}-g_{L} t\right)\right),
$$

which acts as a boundary condition, we follow the characteristics

$$
\left\{\begin{array}{l}
\dot{x}(t)=g_{R} \\
x\left(t_{s}\right)=x_{s}
\end{array}\right.
$$

given by

$$
x(t)=x_{s}+\left(t-t_{s}\right) g_{R}
$$

to reach $x_{s}$, at time

$$
t_{s}=t-\frac{\left(x-x_{s}\right)}{g_{R}} .
$$

Finally, the solution is

$$
\phi(x, t)= \begin{cases}\phi_{0}\left(x-g_{L} t\right) & \text { for } x<x_{s} \\ T r\left(t_{s}\right) \text { for } \quad\left(x_{s}-g_{R} t\right) \leq\left(x-g_{R} t\right)<x_{s} \\ \phi_{0}\left(x-g_{R} t\right) \text { for } \quad\left(x-g_{r} t\right) \geq x_{s}\end{cases}
$$

where $\operatorname{Tr}$ is defined by condition (A.3) and $t_{s}$ is defined by (A.4).

Case with source term. It can be deduced from the homogeneous case by changing the unknowns. In the subregion where $\Lambda$ is constant, if $\phi(x, t)$ is solution of

$$
\partial_{t} \phi+\partial_{x} g \phi=\Lambda \phi
$$

then

$$
\tilde{\phi}(x, t)=e^{\Lambda t} \phi(x, t)
$$

is solution of

$$
\partial_{t} \tilde{\phi}+\partial_{x} g \tilde{\phi}=0
$$

We distinguish two cases:

With a source term in the left subdomain $\Lambda_{L}>0$ and $\Lambda_{R}=0$, the solution is

$$
\phi(x, t)=\left\{\begin{array}{l}
\phi_{0}\left(x-g_{L} t\right) \exp (-\Lambda t) \text { for } x<x_{s} \\
\operatorname{Tr}\left(t_{s}\right) \exp \left(-\Lambda t_{s}\right) \text { for }\left(x_{s}-g_{R} t\right) \leq\left(x-g_{R} t\right)<x_{s}, \\
\phi_{0}\left(x-g_{R} t\right) \text { for }\left(x-g_{R} t\right) \geq x_{s} .
\end{array}\right.
$$

where $\operatorname{Tr}$ is defined by the transmission condition (A.3) and $t_{s}$ is defined by (A.4).

With a source term in the right subdomain $\Lambda_{L}=0$ and $\Lambda_{R}>0$, the solution is

$$
\phi(x, t)=\left\{\begin{array}{l}
\phi_{0}\left(x-g_{L} t\right) \quad \text { for } \quad x<x_{s}, \\
\operatorname{Tr}\left(t_{s}\right) \exp \left(-\Lambda\left(t-t_{s}\right)\right) \text { for }\left(x_{s}-g_{R} t\right) \leq\left(x-g_{R} t\right)<x_{s}, \\
\phi_{0}\left(x-g_{R} t\right) \exp (-\Lambda t) \text { for }\left(x-g_{R} t\right) \geq x_{s} .
\end{array}\right.
$$

where $\operatorname{Tr}$ is defined by the transmission condition (A.3) and $t_{s}$ is defined by (A.4). 
A.2. Exact solution of the 2D problems with piecewise constant speeds.

\section{A.2.1. Horizontal speed.}

Problem.

$$
\left\{\begin{array}{l}
\partial_{t} \phi+\partial_{x} g \phi=0 \quad \text { for }(x, y) \in\left[0, L_{x}\right] \times\left[0, L_{y}\right] \\
g(x, y)= \begin{cases}g_{L} & \text { if } \quad x<x_{s} \\
g_{R} & \text { if } \quad x \geq x_{s}\end{cases} \\
\phi(x, y, 0)=\phi_{0}(x, y) \\
\psi_{L}\left(\phi\left(x_{s}^{-}, y, t\right)\right)=\phi\left(x_{s}^{+}, y, t\right)
\end{array}\right.
$$

Solution. The solution is

$$
\phi(x, y, t)= \begin{cases}\phi_{0}\left(x-g_{L} t, y\right) & \text { for } x<x_{s} \\ \operatorname{Tr}\left(t_{s}, y\right) \text { for } \quad\left(x_{s}-g_{R} t\right) \leq\left(x-g_{R} t\right)<x_{s} \\ \phi_{0}\left(x-g_{R} t, y\right) \text { for }\left(x-g_{R} t\right) \geq x_{s}\end{cases}
$$

where $\operatorname{Tr}$ is defined by the transmission condition

$$
\operatorname{Tr}(t, y)=\psi_{L}\left(\phi_{0}\left(x_{s}-g_{L} t, y\right)\right)
$$

and $t_{s}$ is defined by (A.4).

\section{A.2.2. Shear.}

Problem.

$$
\left\{\begin{array}{lr}
\partial_{t} \phi+\partial_{y} \phi=0 \quad \text { for }(x, y) \in\left[0, L_{x}\right] \times\left[0, y_{s}\right] \\
\partial_{t} \phi+\partial_{x} \phi+\partial_{y} \phi=0 \quad \text { for }(x, y) \in\left[0, L_{x}\right] \times\left[y_{s}, L_{y}\right] \\
\phi(x, y, 0)=\phi_{0}(x, y) & \text { (initial condition) }
\end{array}\right.
$$

Solution. The characteristics are of the form

$$
\left\{\begin{array}{l}
x(t)=x_{0}+t^{D} \\
y(t)=y_{0}+t_{s}+t^{D}
\end{array}\right.
$$

with $t^{D}$ the time spent in the upper zone

$$
t^{D}=y-y_{s}
$$

We can then define the solution piecewise

$$
\phi(x, y, t)=\left\{\begin{array}{l}
\phi_{0}\left(x-t^{D}, y-t\right) \quad \text { for }(x, y) \in\left[0, L_{x}\right] \times\left[y_{s}, L_{y}\right] \\
\phi_{0}(x, y-t) \quad \text { for }(x, y) \in\left[0, L_{x}\right] \times\left[0, y_{s}\right]
\end{array}\right.
$$

\section{A.2.3. Waterproof.}


Problem.

$$
\left\{\begin{array}{l}
\partial_{t} \phi+\partial_{x} \phi=0 \quad \text { for }(x, y) \in\left[x_{I}, L_{x}\right] \times\left[0, y_{s}\right] \\
\partial_{t} \phi+\partial_{x} \phi+\partial_{y} \phi=0 \quad \text { for }(x, y) \in\left[0, L_{x}\right] \times\left[0, L_{y}\right]-\left[x_{I}, L_{x}\right] \times\left[0, y_{s}\right] \\
\phi(x, y, 0)=\phi_{0}(x, y)
\end{array}\right.
$$

In order to close this problem, we have to add a homogeneous Dirichlet condition on the north of the limit between the 2 zones

$$
\phi\left(x, y_{s}^{+}, t\right)=0 \quad \text { for } x \in\left[x_{s}, L_{x}\right]
$$

Solution. As in the precedent case the characteristics are of the form

$$
\left\{\begin{array}{l}
x(t)=x_{0}+t^{D}, \\
y(t)=y_{0}+t_{s}+t^{D} .
\end{array}\right.
$$

We can then define the solution piecewise

$$
\phi(x, y, t)=\left\{\begin{array}{l}
\phi_{0}(x-t, y-t) \quad \text { for }(x, y) \in\left[0, L_{x}\right] \times\left[y_{s}, L_{y}\right] \quad \text { and }\left(x-t^{D}\right) \leq x_{s} \\
0 \quad \text { for }(x, y) \in\left[0, L_{x}\right] \times\left[y_{s}, L_{y}\right] \quad \text { and }\left(x-t^{D}\right)>x_{s} \\
\phi_{0}(x-t, y-t) \quad \text { for }(x, y) \in \Omega_{1} \\
\phi_{0}\left(x-t, y-t_{s}\right) \quad \text { for }(x, y) \in \Omega_{2}
\end{array}\right.
$$



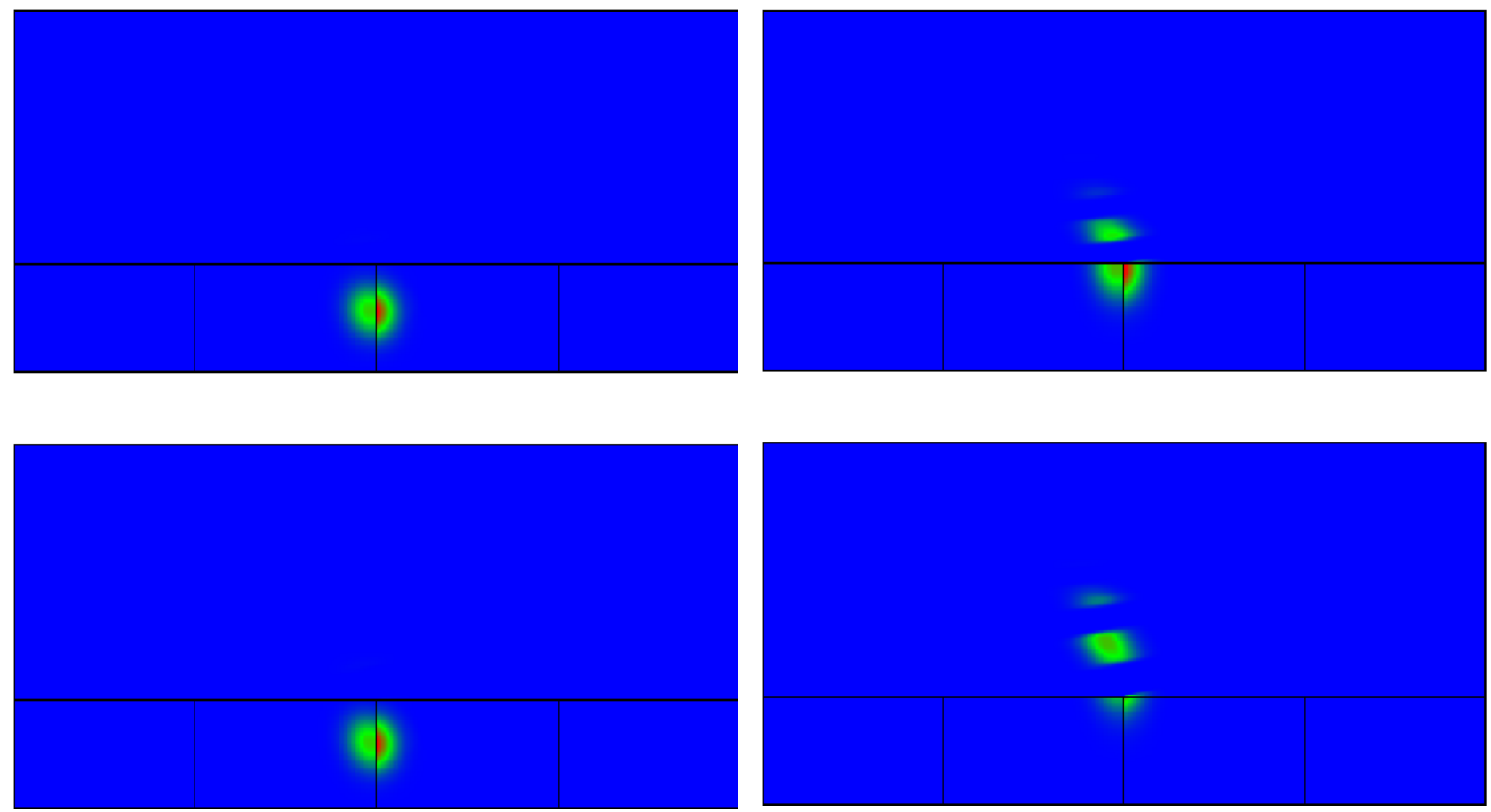

a) $t=0.66$, cell cycles $1-2$ transition $\Omega_{2}^{1} \rightarrow \Omega_{1}^{2}$. $\phi_{\max }=140$

b) $t=2.58$, cell cycles $3-4$ transition $\Omega_{2}^{3} \rightarrow \Omega_{1}^{4}$ and $\Omega_{1}^{4} \nearrow \Omega_{3}$, $\phi_{\max }=430$
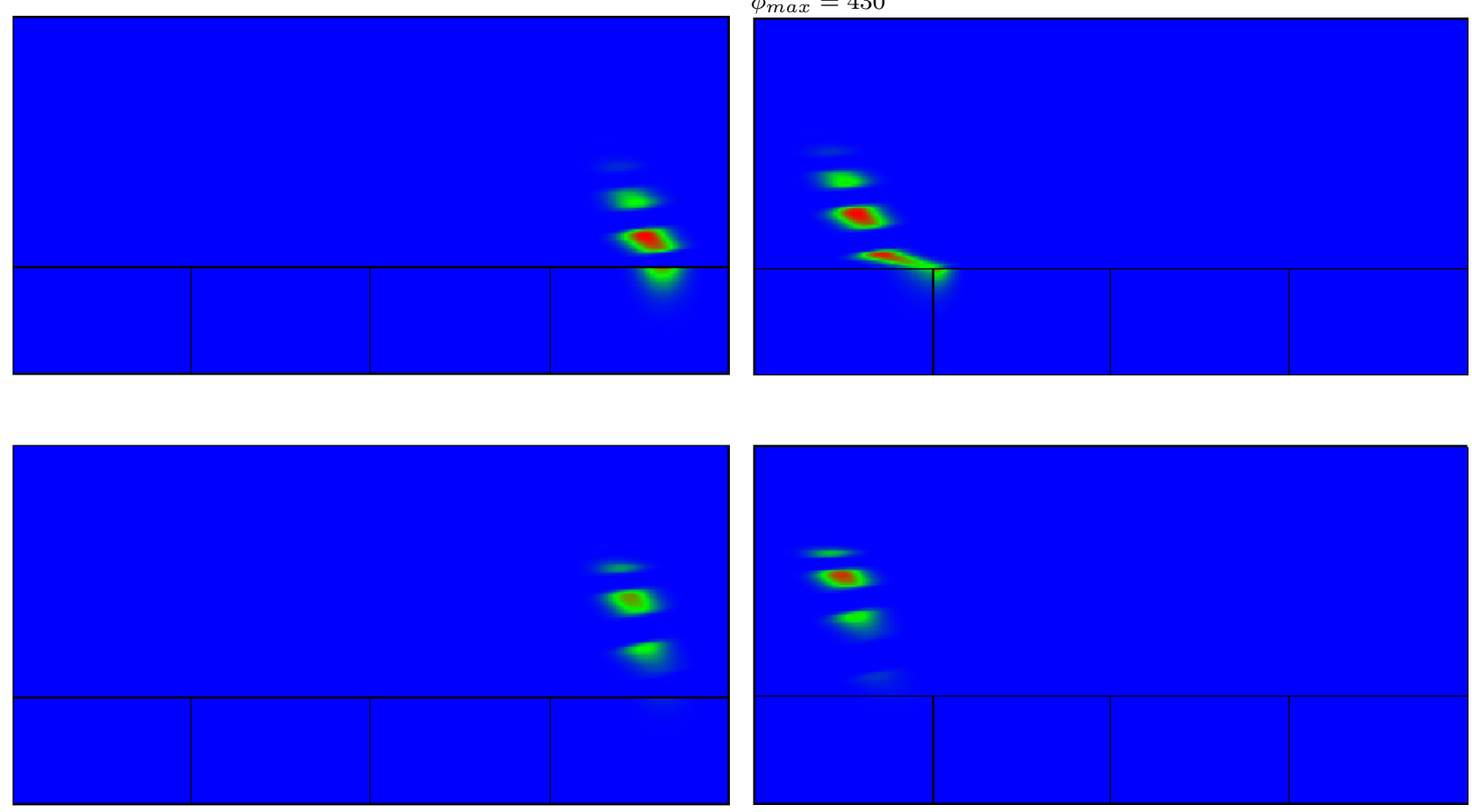

c) $t=3.35$, cell cycles $3-4$, waterproof boundary between $\Omega_{2}^{4}$ and $\Omega_{3}$ in the 4 th cell cycle for follicle $1, \phi_{\max }=495$

d) $t=3.8$, cell cycles $5-6$, differentiation in $\Omega_{3}$, residual mass in cycle $\Omega_{2}^{5}$ for follicle $1, \phi_{\max }=510$

FIGURE 5.3. Full model: snapshots of the density at different times of the follicular development, $\Delta x=0.0125$. The color code is time related. Blue and red colors indicate respectively null and maximum observed density $\phi_{\max }$ over the whole domain at the snapshot times. Follicle 1 (top) and follicle 2 (bottom) differ by $\tau_{h}=0.7$ and 1.0 respectively 

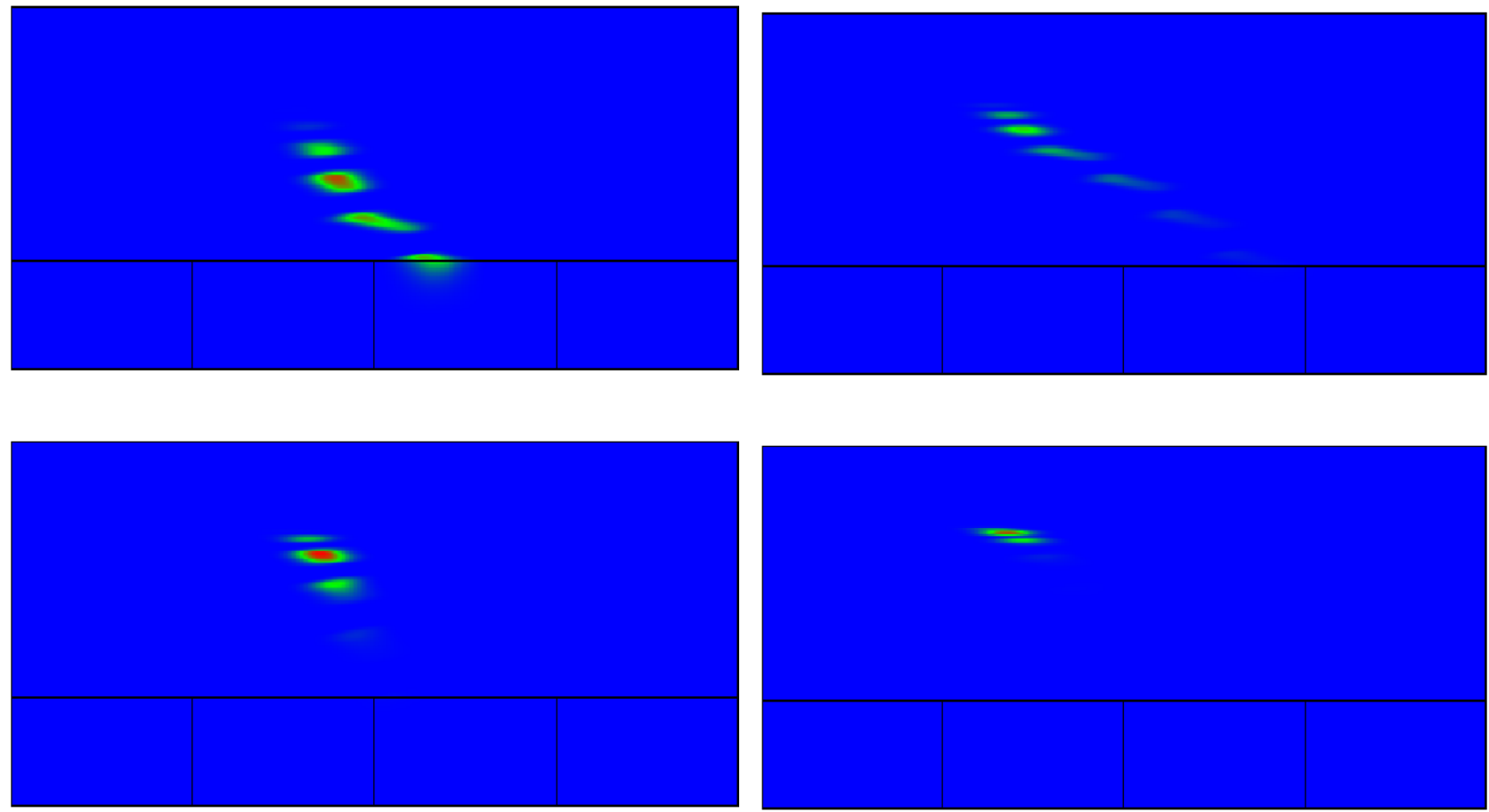

a) $t=4.48$, cell cycles $5-6$, differentiation in $\Omega_{3}$, residual mass

b) $t=6.30$, cell cycles $7-8$, differentiation in $\Omega_{3}, \phi_{\max }=1600$ in the 6th cycle $\Omega_{1}^{6}$ for follicle $1, \phi_{\max }=660$

FIGURE 5.4. Full model: snapshots of the density at different times of the follicular development, $\Delta x=0.0125$. The color code is time related. Blue and red colors indicate respectively null and maximum observed density $\phi_{\max }$ over the whole domain at the snapshot times. Follicle 1 (top) and follicle 2 (bottom) differ by $\tau_{h}=0.7$ and 1.0 respectively

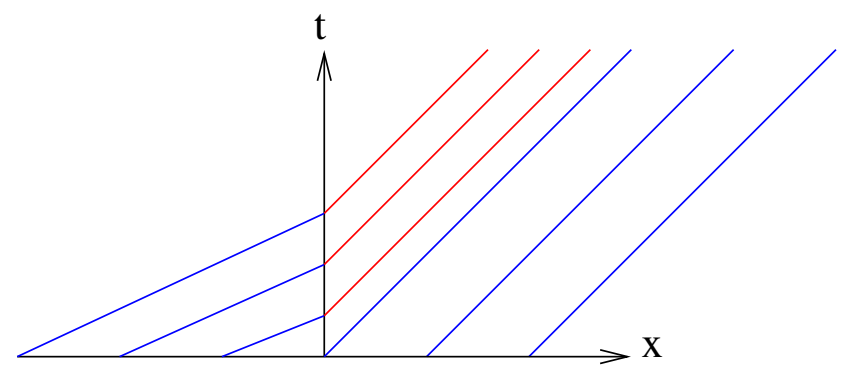

FIGURE A.1. Characteristics of a transmission problem with a transmission condition. The solution is a stationnary shock, corresponding to $g_{R}<g_{L} \psi_{L}^{\prime}$. 